\title{
Modelling of Hybrid Scenario: from present-day experiments toward ITER
}

\author{
X. Litaudon ${ }^{1}$, I. Voitsekhovitch ${ }^{2}$, J.F. Artaud ${ }^{1}$, P. Belo ${ }^{3}$, João P.S. Bizarro ${ }^{3}$, T Casper ${ }^{4}$, \\ J. Citrin ${ }^{5}$, E. Fable ${ }^{6}$, J. Ferreira ${ }^{3}$, J. Garcia ${ }^{1}$, L. Garzotti ${ }^{2}$, G. Giruzzi ${ }^{1}$, J. Hobirk ${ }^{6}$, \\ G.M.D. Hogeweij ${ }^{5}$, F. Imbeaux ${ }^{1}$, E. Joffrin ${ }^{1}$, F. Koechl ${ }^{7}$, F. Liu ${ }^{1}$, J. Lönnroth ${ }^{8}$, D. Moreau ${ }^{1}$, V. \\ Parail $^{2}$, M. Schneider ${ }^{1}$, P.B. Snyder ${ }^{9}$, ASDEX-Upgrade Team, JET-EFDA contributors ${ }^{*}$, and \\ the EU-ITM ITER Scenario Modelling group
}

\author{
${ }^{1}$ CEA, IRFM, F-13108 Saint Paul Lez Durance, France \\ ${ }^{2}$ EURATOM/CCFE Fusion Association, Culham Science Centre, Abingdon OX14 3DB UK \\ ${ }^{3}$ Associação Euratom-IST, Instituto de Plasmas e Fusão Nuclear, Instituto Superior Técnico, \\ Universidade Técnica de Lisboa, 1049-001 Lisboa, Portugal \\ ${ }^{4}$ ITER Organization, F-13115 Saint Paul lez Durance, France \\ ${ }^{5}$ FOM Institute DIFFER - Dutch Institute for Fundamental Energy Research, Association \\ EURATOM-FOM, Nieuwegein, The Netherlands \\ ${ }^{6}$ Max-Planck-Institut für Plasmaphysik, EURATOM-Assoziation, Garching, Germany \\ ${ }^{7}$ Association EURATOM-ÖAW/ATI, Atominstitut, TU Wien, 1020 Vienna, Austria \\ ${ }^{8}$ Helsinki University of Technology, Association EURATOM-Tekes, P.O.Box 4100, FIN- \\ 02015 TKK, Finland \\ ${ }^{9}$ General Atomics, San Diego, USA
}

e-mail of first author: xavier.litaudon@cea.fr

\begin{abstract}
The 'hybrid' scenario is an attractive operating scenario for ITER since it combines long plasma duration with the reliability of the reference H-mode regime. We review the recent European modelling effort carried out within the Integrated Scenario Modelling group which aims at (i) understanding the underlying physics of the hybrid regime in ASDEX-Upgrade and JET, and, (ii) extrapolating them toward ITER. JET and ASDEX-Upgrade hybrid scenarios performed under different experimental conditions have been simulated in an interpretative and predictive way in order to address the current profile dynamics and its link with core confinement, the relative importance of magnetic shear, $s$, and $E x B$ flow shear on the core turbulence, pedestal stability and H-L transition. The correlation of the improved confinement with an increased $s / q$ at outer radii observed in JET and ASDEX-Upgrade discharges is consistent with the predictions based on the GLF23 model applied in the simulations of the ion and electron kinetic profiles. Projections to ITER hybrid scenarios have been carried out focusing on optimization of the heating/current drive schemes to reach and ultimately control the desired plasma equilibrium using ITER actuators. Firstly, access condition to the hybrid-like q-profiles during the current ramp-up phase has been investigated. Secondly, from the interpreted role of the $s / q$ ratio, ITER hybrid scenario flat-top performance has been optimized through tailoring the q-profile shape and pedestal conditions. EPED predictions of pedestal pressure and width have been used as constraints in the interpretative modelling while the core heat transport is predicted by GLF23. Finally, model based approach for real-time control of advanced tokamak scenarios has been applied to ITER hybrid regime for simultaneous magnetic and kinetic profile control.
\end{abstract}

\footnotetext{
* See the Appendix of F. Romanelli et al., Proceedings of the 24th IAEA Fusion Energy Conference 2012, San Diego, USA
} 


\section{Introduction}

An attractive operating scenario for ITER has been proposed and reviewed for instance in [1] that combines long plasma duration similar to the steady-state scenario, together with the reliability of the reference $\mathrm{H}$-mode regime. The so-called 'hybrid' scenario aims to maximize neutron fluence in a reliable manner for engineering tests with an extended burn time $(\mathrm{t}>1000 \mathrm{~s})$ together with significant fusion gain, $\mathrm{Q} \geq 5$ [2-3]. To achieve this goal, the plasma current in this scenario is lower (12-14 MA) than the reference H-mode scenario but higher than steady state scenarios. From the engineer point of view this is an intermediate operating point between the H-mode and the steady-state scenarios where the plasma current is driven by a combination of inductive and non-inductive currents with moderate assumption on confinement and beta as defined in reference [2]. Worldwide a significant experimental effort has been devoted to explore the operating space in present day tokamaks. When reducing the plasma current and with modified plasma current density profile, it was found in present experiment that the confinement could be increased above the reference H-mode scenario which would allow operating ITER with a high fusion gain $(\mathrm{Q} \sim 10)$ for long pulse duration. This paper is an overview of the recent European modelling effort carried out within the Integrated Scenario Modelling working group (ISM-WG) which aims at (i) understanding the underlying physics of the hybrid regime in ASDEX-Upgrade and JET, and, (ii) extrapolating them toward ITER. The ISM-WG is organized within the European Fusion Development Agreement (EFDA) Task Force on Integrated Tokamak Modelling (ITM-TF) [4-5]. The main responsibility of the ISM-WG is to advance a pan-European approach to (i) interpretative modelling of existing experiments to validate and benchmark integrated modelling tools and (ii) to predictive modelling of JT-60SA [6] and ITER [7] plasmas with the emphasis on urgent issues.

In this paper, plasma current density evolution, heat, particle and momentum transport, and pedestal characteristics in JET and ASDEX-Upgrade hybrid discharges are investigated by means of various integrated modelling tools (ASTRA [8], CRONOS [9], JETTO [10]). Predictions of ITER hybrid scenarios are then carried out making use of the findings obtained from the analysis of existing experiments. Since it is not possible to reproduce all the physics parameters of ITER plasmas simultaneously in present experiments, simulations are used to project to the ITER regime using theoretically based physics models that are being tested against present tokamak experiments. The important contributions of our approach consists in using two experimental devices (JET and ASDEX-U) to validate the integrated modelling of various phases of the hybrid scenario and for the extrapolation to ITER hybrid scenario first principle modelling is used to predict both the core and pedestal confinement. This paper complements (i) previous European studies performed within the ISM-WG focusing on the ITER baseline scenario [6], (ii) the international effort coordinated by the Integrated Operation Scenario (IOS) topical group of the International Tokamak Physics Activity (ITPA) to compare the various codes prediction for the hybrid and steady-state scenarios $[11,12]$, (iii) previous studies such as [13] and (iv) finally the most recent ITER predictive modelling of three main scenarios performed within an F4E grant [14].

After this introduction, the paper is organized in two main sections. In section 2, recent integrated modelling of the JET and ASDEX-Upgrade hybrid scenario is discussed focusing on the neo-classical current diffusion issues, on the importance of the q-profile on core confinement, on the self-consistent modelling of thermal, particle and momentum transport using first principle transport models and finally on the modelling of the scenario termination 
when ramping down the power and current. In section 3, extrapolation of our validation exercise on existing experiment to ITER hybrid scenario is performed with the ITER baseline heating and current drive mix. The operational domain of the ITER hybrid scenario is first estimated using assumption made from 0-D scaling. Then the possibility to access to the hybrid type of q-profile during the ramp-up phase is extensively studied. During the ITER burn phase, first principle calculations will be presented in details in view of predicting simultaneously the pedestal and the core performance. Finally before the conclusion, the ability to control in real time simultaneously the magnetic and kinetic profiles will be discussed. 


\section{Integrated modeling of ASDEX-Upgrade and JET hybrid scenario}

More than fourteen JET and two ASDEX-Upgrade hybrid scenarios performed under different experimental conditions (plasma shape, heating power, plasma current ramp-up waveform, dimensionless parameters etc.) have been simulated in an interpretative and predictive way in order to address the current profile dynamics and its link with (improved) confinement, the relative importance of magnetic shear, $s$, and radial electric, $E x B$, flow shear on the core turbulence, pedestal stability and H-L transition. The capability of the transport models to predict the plasma evolution during the whole scenario (current ramp-up, main heating phase and current ramp-down) is examined in this section.

For both machines, a variation in q-profile at the start of the main heating phase was experimentally achieved but using different techniques as illustrated on Fig. 1. By optimising the current density profile (i.e. broadening the current profile with flat core q profile over a large part of the plasma radius), enhanced confinement factor, $H_{I P B 98(y, 2)}$, with respect to the IPB98(y,2) scaling [15] have been observed up to levels of 1.4. For JET, this variation was achieved via the 'current-overshoot' method (e.g. \#77922, \#79626 compared to \#79630 without overshoot) [16-18]. With this method, the current is ramped down to its flattop value just prior to the main heating phase, resulting in a broader q-profile compared with a regular ramp-up scenario. This technique has been first applied successfully to low triangularity $(\delta=0.2)$ at low magnetic field strength $\left(B_{T}=1.7 T / I_{p}=1.4 M A\right)$ and densities of the order of $50 \%$ of the Greenwald density $n_{G w}\left(n_{G w}=I_{P} / \pi a^{2}\right)$. It has then be applied to high triangularity ITERlike shape $(\delta \sim 0.4)$ and thus to higher density (75\% of the Greenwald density) with $H_{I P B 98(y, 2)}=1.3-1.4$ and $\beta_{\mathrm{N}} \sim 3$. These results have been extended to higher field $\left(B_{T}=2.3 T\right)$ and current (up to 2MA). Finally, performance of the hybrid regime has been extended toward long pulse discharge on JET up to 20s (\#77280) and maintained for duration of typically two resistive times. For ASDEX-Upgrade [19], the q-profile modification was achieved by varying the auxiliary heating timing, with the later heating case (e.g. \#20995 compared to \#20993) resulting in a broader q-profile with different MHD-behaviour (the early heating scheme triggers early $(4,3)$ - or $(3,2)$-NTMs, whereas the late-heating scheme, pulse \#20995, shows (1,1)-fishbones).. Table 1 summarises the typical range of parameters for a selection of the most representative JET discharges and the two ASDEX-Upgrade discharges. It indicates also the domain of dimensionless parameters cover by these two experiments that complement each other when extrapolating our modelling results toward ITER. 
Table 1: O-D parameters of JET and ASDEX-Upgrade hybrid scenario during the high beta phase when the fusion performance (e.g. neutron yield) is maximum. Major radius, $R_{o}$, minor radius, $a$, elongation, $k$, triangularity, $\delta$, on axis toroidal magnetic field, $B_{T}$, plasma current $I_{p}$, safety factor at 95\% of the poloidal flux, $q_{95}$, applied power, $P_{\text {tot }}$, the thermal confinement factor $H_{I P B 98(y, 2)}$, the normalised total pressure, $\beta_{N}$ (from diamagnetic measurements), the core and volume averaged electron density, $n_{e o},\left\langle n_{e}\right\rangle$, the volume averaged ion and electron temperature $\left\langle T_{i}\right\rangle,\left\langle T_{e}\right\rangle$. The dimensionless quantities: the ion Larmor radius, the effective electron collision frequency the toroidal Mach number $\left(\rho_{i}^{*}, v_{e}^{*}, M_{\Phi}\right)$.

\begin{tabular}{|c|c|c|c|c|c|c|c|}
\hline & \multicolumn{5}{|c|}{ JET } & \multicolumn{2}{|c|}{ ASDEX-Upgrade } \\
\hline & 77922 & 79626 & 79630* & 79635 & 77280 & $20993 *$ & 20995 \\
\hline$R_{o}[m], a[m]$ & $3.1,0.9$ & $3.1,0.9$ & $3.1,0.9$ & $3.1,0.9$ & $3.1,0.9$ & $1.61,0.51$ & $1.6,0.51$ \\
\hline$k, \delta$ & $1.7,0.4$ & $1.6,0.2$ & $1.6,0.2$ & $1.7,0.3$ & $1.7,0.4$ & $1.73,0.24$ & $1.75,0.24$ \\
\hline$B_{T}[T]$ & 2.3 & 2 & 2 & 1.4 & 1.7 & 2.34 & 2.34 \\
\hline$I_{p}[M A]$ & 1.7 & 1.7 & 1.7 & 0.9 & 1.1 & 1.0 & 1.0 \\
\hline$q_{95}$ & 4.2 & 3.9 & 3.9 & 4.8 & 4.9 & 4.6 & 4.65 \\
\hline$P_{t o t}[M W]$ & 18 & 17 & 17 & 7 & 10 & 8 & 8 \\
\hline$H_{I P B 98(y, 2)}$ & 1.3 & 1.3 & 1.1 & 1.3 & 1.25 & 1.0 & 1.2 \\
\hline$\beta_{N}$ & 2.8 & 2.8 & 2.6 & 2.4 & 2.5 & 2 & 2.3 \\
\hline$n_{e o},\left\langle n_{e}\right\rangle$ & $5.5,3.9$ & $4.6,2.7$ & $4.3,2.7$ & $2.9,2.1$ & $3.7,2.6$ & $6.1,4.8$ & $6.9,4.9$ \\
\hline$\left\langle\boldsymbol{T}_{i}\right\rangle,\left\langle\boldsymbol{T}_{e}\right\rangle$ & $3.2,2.4$ & $3.6,2.3$ & $3.5,2.2$ & $1.3,1.1$ & $2.1,1.6$ & $2.0,1.7$ & $2.3,1.9$ \\
\hline$\rho *_{i} \times 10^{-3}$ & 3.9 & 4.8 & 4.7 & 4.1 & 4.3 & 5.4 & 5.8 \\
\hline$v *_{e}$ & 0.13 & 0.09 & 0.1 & 0.37 & 0.2 & 0.15 & 0.13 \\
\hline$M_{\Phi}$ & 0.5 & 0.5 & 0.6 & 0.8 & 0.4 & 0.4 & 0.5 \\
\hline
\end{tabular}

*reference H-mode discharges with the engineer parameters (e.g. plasma current, toroidal magnetic field etc.) set as for the hybrid scenario 


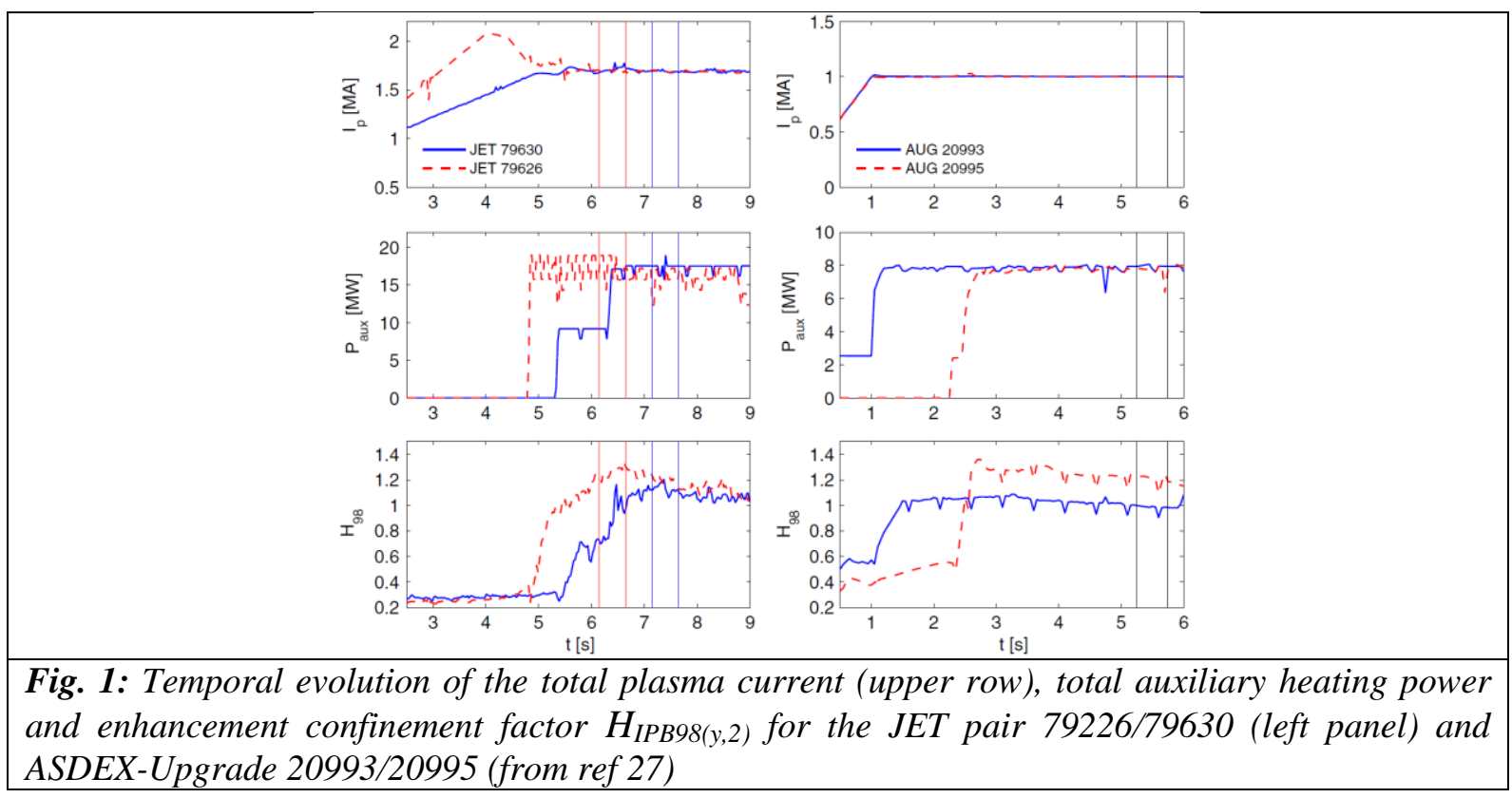

\subsection{Current diffusion}

Current diffusion using neo-classical prediction for the resistivity and bootstrap current is simulated for JET and ASDEX-Upgrade with the CRONOS code [8] by doing an interpretative analysis [20]. The neoclassical quantities are deduced from the NCLASS code [20]. NCLASS solves the flux-surface-averaged parallel momentum and heat-flow balance equations for each plasma species using the formulation of Hirshman and Sigmar [22]. The velocity-dependent viscosity matrices are taken from a publication by Shaing et al [23] and are valid in all collision regimes and aspect ratios. The same modelling assumptions have been made for JET and ASDEX-Upgrade. The simulations are initiated at the time when the first MSE data are available, i.e. usually just after the NBI application. As a consequence, the initial magnetic equilibrium is prescribed by the first q-profile determined by the magnetic reconstruction constrained by MSE measurement which, in the case of ASDEX Upgrade, is performed by the CLISTE code [24] and in the case of JET by EFIT code [25]. The simulated q-profiles with CRONOS using the measured kinetic profiles (temperature and density) are then compared at each time step to the other MSE measurements available for each discharge. The effective charge profiles are provided by the charge exchange recombination spectroscopy measurement for JET and from the deconvolution of the bremsstrahlung measurement for ASDEX-Upgrade. In JET hybrid discharges, two interpretative analysis are illustrated in this paper, one for the discharge \#77922, which last for one current diffusion time, and another one for the shot \#77280, which last for 2 current diffusion times (20s long hybrid discharge). The current profile slowly relaxes after the $\mathrm{H}$-mode transition with on-axis $\mathrm{q}_{\mathrm{o}} \sim 1$ and its dynamics is reasonably well reproduced with the neo-classical approximation as shown in figure 2. Conversely, for ASDEX-Upgrade it is found that the q-profile is rapidly clamped to the $\mathrm{q}_{\mathrm{o}}=1$ surface in the studied discharge \#20995 with $(1,1)$-fishbones activity while neo-classical current diffusion simulation predicts a slow relaxation with $\mathrm{q}_{\mathrm{o}}$ below unity. For ASDEX-Upgrade a similar discrepancy between the measurement and simulation was reported in [26] with the ASTRA code used in an interpretive manner but on a different discharge. The reasons for the differences in the current profile relaxation between JET and ASDEX-Upgrade will be examined in the future in particular by investigating the subtle differences between the two experiments. 


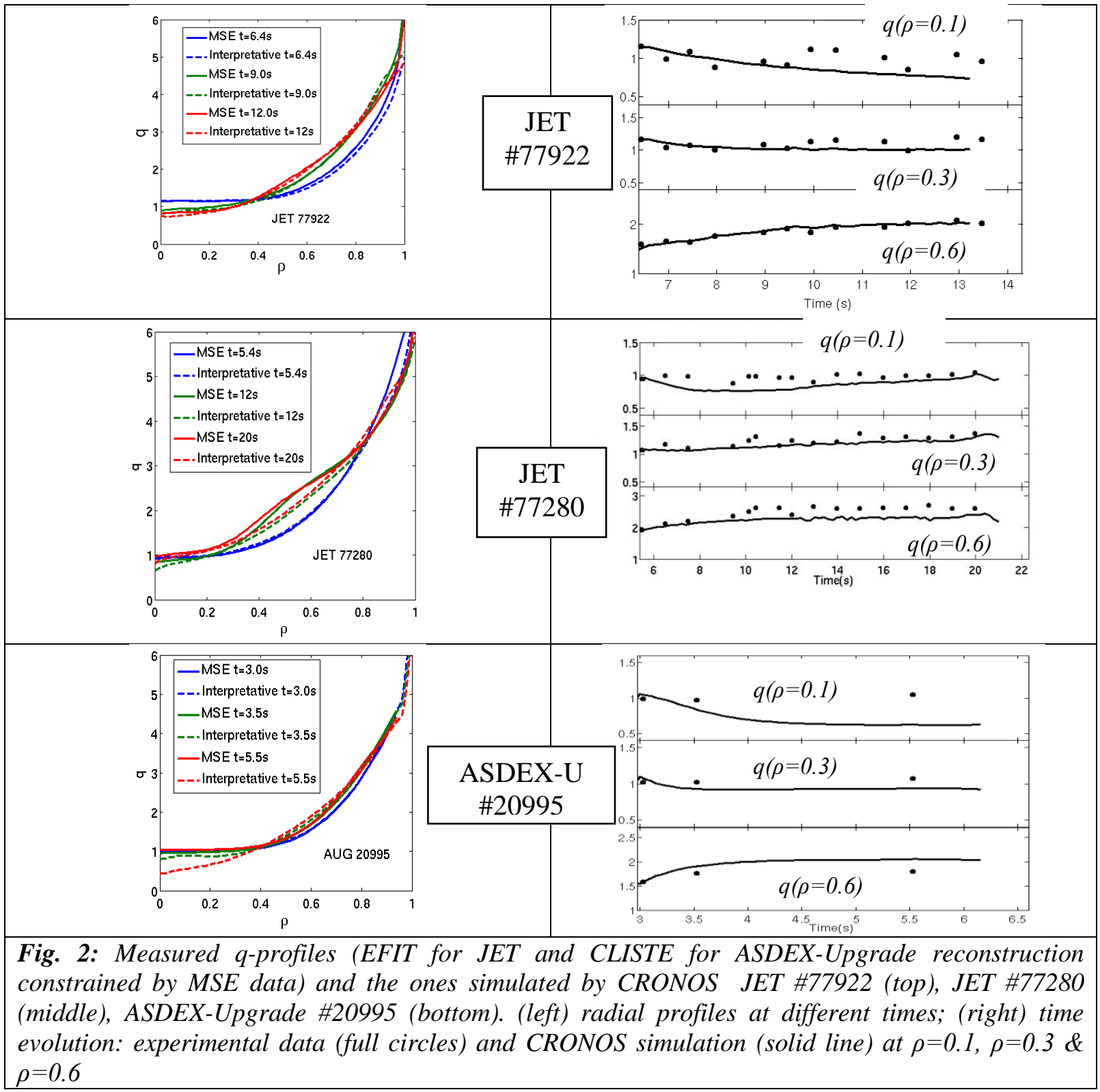

\section{2 q-profile influence on transport}

In certain experimental conditions, hybrid scenarios are characterized by improved thermal confinement compared to the H-mode empirical scaling law expectations (i.e. IPB98(y,2)). Modelling effort is carried out to isolate the impact of increased $s / q$ at outer radii (where $s$ is the magnetic shear) on core confinement in low-triangularity JET and ASDEX-Upgrade experiments [27]. Predictive heat and particle transport is calculated using the integrated modelling code CRONOS coupled to the GLF23 turbulent transport model [28]. For both machines, discharge pairs were analysed displaying similar pedestal confinement yet significant differences in core confinement. Therefore, this approach complements previous studies [26] where the global confinement enhancement was interpreted by an improved pedestal pressure in pair of discharges where the core confinement was similar. In this proposed study, the focus is on the core confinement analysis for similar pedestal confinement but we do not exclude that both core and pedestal enhancement may explain, depending on the experimental conditions, the increase of confinement in the hybrid scenario. For the JET 
pair (\#79626/\#79630), this variation was achieved via the 'current-overshoot' method $[17,18]$ (c.f. Fig. 1) . After a fast ramp-up, the current is ramped down to its flat-top value just prior to the main heating phase resulting in a transient broader q-profile with improved confinement (\#79626 with $\mathrm{H}_{\mathrm{IPB} 98(\mathrm{y}, 2)}$ 1.3) compared with a regular ramp-up scenario (\#79630 with

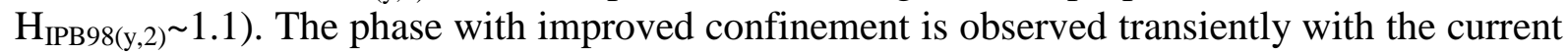
overshoot technique. For the ASDEX-Upgrade pair (\#20993/\#20995), the q-profile variation was achieved by varying the auxiliary heating timing, with the later heating case resulting in a broader q-profile with improved confinement (\#20995, $\left.\mathrm{H}_{\mathrm{IPB} 98(\mathrm{y}, 2)} \sim 1.2\right)$ compared to the reference case (\#20993, $\mathrm{H}_{\mathrm{IPB} 98(\mathrm{y}, 2)}$ 1.0) [19] (c.f. Fig. 1). The $s / q$ profiles for the JET and ASDEX-Upgrade discharges indicate an increase of $s / q$ in the region $\rho \sim 0.4-0.8$ of more than $20 \%$ (and a reduction of $s / q$ inside $\rho \sim 0.4$ ) for the discharges with optimised q-profile for confinement.

The main objective of the work discussed in this section is to determine the importance of the q-profile on the core confinement. To this end, simulations for the improved confinement cases (either JET 79630 or ASDEX-U 20995) were carried out substituting the q-profile input with the q-profile corresponding to the partner discharge in each pair.. In such a manner GLF23 predicts the confinement difference solely due to the q-profile. To illustrate such modelling, Fig. 3 displays results of ion temperature transport simulation with GLF23 model (without ExB shear stabilisation) for the JET and ASDEX-Upgrade comparing results with qprofile inputs taken from either the low or high confinement discharges. This approach allows isolating the role of the q-profile by changing only this quantity. For both devices, simulations with the q-profile corresponding to the improved confinement case display improved ion confinement compared with a simulation which is identical apart from substituting in the qprofile (Fig. 3). It was concluded from a full set of modelling (with or without ExB stabilisation, with or without particle transport etc), that this effect accounts for $\sim 60-90 \%$ and $\sim 35-55 \%$ of the core confinement improvement in JET and ASDEX-Upgrade respectively. These results are consistent with an increase of the ITG threshold with $s / q$. Correlation of the improved confinement with an increased $s / q$ at outer radii observed in low triangularity JET and ASDEX-Upgrade discharges is consistent with the predictions based on the GLF23 model The successful prediction of the core energy differences due to the $s / q$ effect, regardless of the $E x B$ model, provides an encouraging validation of the impact of q-profile shaping on core confinement and this effect should be included in ITER modelling. In this section, we have isolated the importance of the s/q parameter in the confinement region at the outer radii. In addition, it is worth mentioning that low magnetic shear in the very core of the discharge combined with the high rotational shear lead to a reduction of the ion stiffness as observed on JET [29]. The impact of the rotation on the ion stiffness remains an open issue on the theory and modelling sides. It deserves further work that is beyond the scope of this paper where established models have been used for the modelling. 


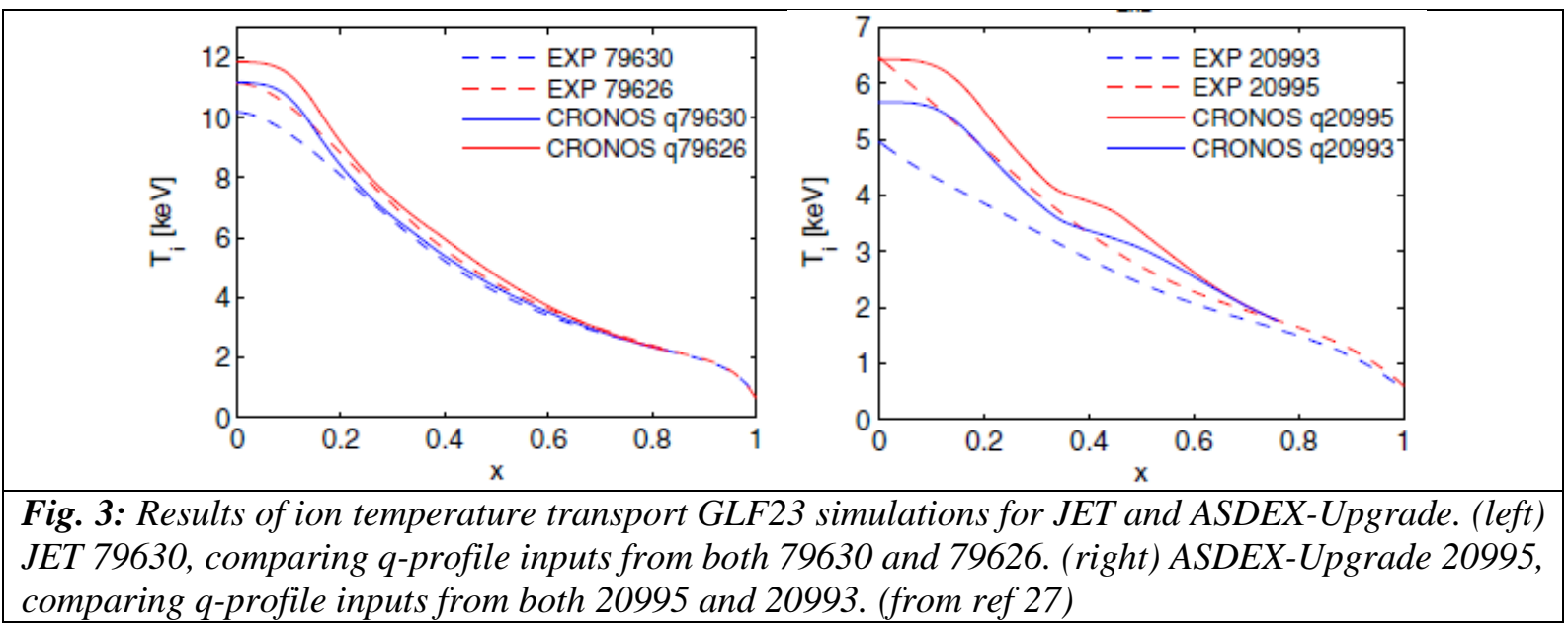

\subsection{Self-consistent current, thermal, particle and rotation modelling of hybrid scenario: $E x B$ shear influence on transport}

Self-consistent four-fields simulations [30] predicting the electron $\left(T_{e}\right)$ and ion $\left(T_{i}\right)$ temperatures, main ion density $\left(n_{i}\right)$ and toroidal angular frequency $(\omega)$ have been performed for eight JET pulses (\#74641, \#74634, \#74637, \#74826, \#75225, \#79635, \#75590, \#77922) by using the GLF23 model in ASTRA code [8]. Four low $\delta$ hybrid pulses (\#74641, \#74634, \#74637, \#75225) are performed at the same magnetic field (2 T), plasma current (1.7 MA) and central line averaged density $\left(n_{l}=3-3.4 \times 10^{19} \mathrm{~m}^{-3}\right)$, but different NBI heating power (9.319 MW) while three high $\delta$ hybrid pulses (\#79635, \#75590, \#77922) are performed at different magnetic field, plasma current, NBI power and electron density $\left(I_{p}=0.8-1.7 \mathrm{MA}\right.$, $B_{0}=1.1-2.3 \mathrm{~T}, P_{N B I}=6-17 \mathrm{MW}, n_{l}=0.5-4.8 \times 10^{19} \mathrm{~m}^{-3}$ ). In addition a low triangularity $\mathrm{H}$-mode pulse \#74826 without plasma current overshoot, but with otherwise similar scenario to one of the hybrid pulses (\#75225) [18] has been simulated. The $H_{I P B 98(y, 2)}$ factor varies from 1 to 1.37 in selected pulses. The core toroidal angular frequency varies by a factor of two from 60 $\mathrm{krad} / \mathrm{s}$ to $137 \mathrm{krad} / \mathrm{s}$. Therefore, this database has been used for the validation of the GLF23 model addressing in particular to the stabilising effect of the $E x B$ shear on the confinement improvement in hybrid scenario.

The NBI heat, particle and momentum sources used in the predictive simulations have been calculated with NUBEAM/TRANSP, while the deuterium neutral influx through the separatrix (wall particle source) has been estimated in the self-consistent TRANSP-EDGE2D simulations. In these simulations the electron and ion heat fluxes through the separatrix and NBI contribution to the deuterium particle flux calculated by TRANSP have been used as an input to EDGE2D while the neutral influx from SOL to plasma has been returned to TRANSP and used for the NBI simulations and estimation of the particle confinement time. The TRANSP-EDGE2D simulations have been done for two selected discharges performed at low (6 MW, \#79635) and high (17 MW, \#77922) NBI power and extrapolated to other pulses.

The GLF23 model applied with the ExB shear calibration factor $\alpha_{E}=1$ (with $\alpha_{E}=\gamma_{\max } / \gamma_{E}$, here $\gamma_{\max }$ is maximum linear growth rate without $E x B$ shear, $\gamma_{E}$ is the $E x B$ shear rate), which gives a satisfactory temperature prediction for the JET H-mode plasmas and high $\beta_{N}$ scenarios [31], strongly under-predicts the particle and momentum transport leading to the over-predicted density and toroidal rotation while $T_{e}$ and $T_{i}$ are in a relatively good agreement with measurements (Fig. 4, left). By reducing the ExB shear strength in the GLF23 model by factor 2 (i.e. $\alpha_{E}=0.5$ ) a more accurate density prediction has been achieved while the simulated 
temperature and rotation were weakly affected by the reduction of $\alpha_{E}$. To improve the prediction of toroidal rotation, these simulations have been repeated assuming that the momentum diffusivity $\chi_{\varphi}$ is a fraction of the thermal ion diffusivity $\chi_{i}$ where $\chi_{i}$ has been computed with the GLF23 model. The Prandtl number $P_{r}=\chi_{\phi} \chi_{i}$ has been adjusted to match the measured toroidal rotation. With this adjustment $\left(P_{r}=0.3\right.$ and 0.5 for low and high $\delta$ pulses correspondingly) an essential improvement in the prediction of the toroidal rotation has been achieved while the density and temperatures remains within 20\% deviation from the measurements (Fig. 4 right and Fig. 5). It should be mentioned that the value of $\alpha_{E}$ found in the self-consistent four-fields GLF23 simulations of JET Hybrid scenario is in agreement with the nonlinear GYRO simulations which shows, that when the destabilizing effect of parallel velocity shear is not included in the ITG turbulence growth rate simulation, the electron and ion transport is quenched near $\gamma_{E} / \gamma_{\max } \approx 2\left(\alpha_{E} \approx 0.5\right)$ [32].

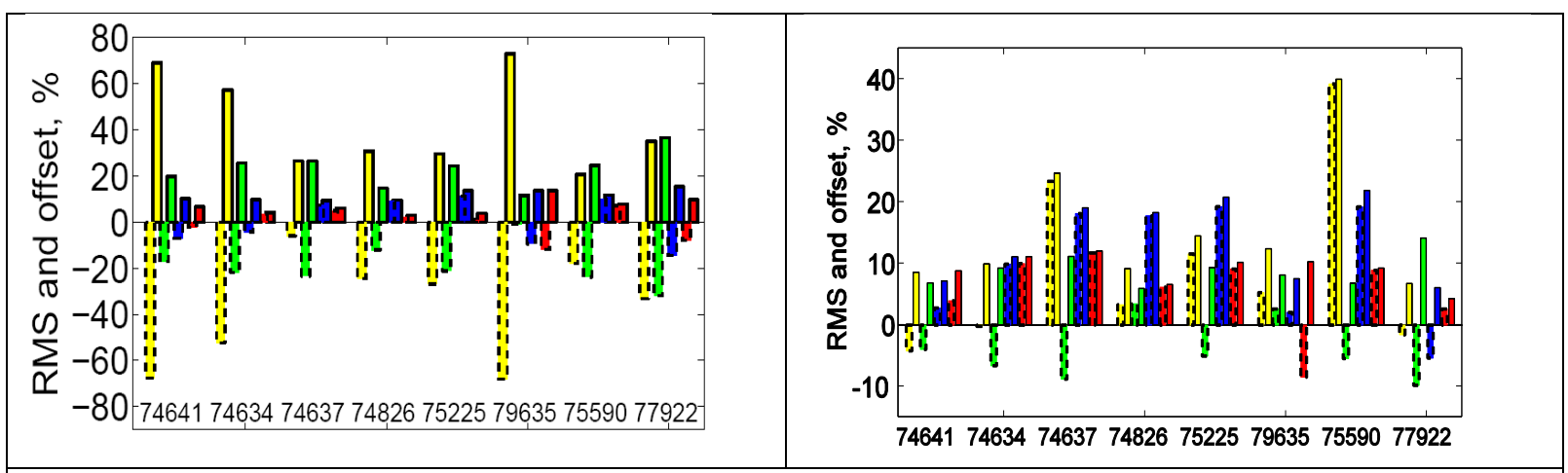

Fig. 4: RMS (solid contour bars) and offset (dashed contour bars) estimated for $T_{e}$ (red), $T_{i}$ (blue), $n_{D}$ (green) and $\omega$ (yellow) using $\alpha_{E}=1$ and GLF23 computed $\chi_{\varphi}\left(\right.$ left), and $\alpha_{E}=0.5$ and $\chi_{\varphi}=P r \cdot \chi_{i}$ with $\operatorname{Pr}=0.3$ (low triangularity) and 0.5 (high triangularity) discharges (right). The H-mode pulse 74826 has been simulated using $\alpha_{E}=1$. 


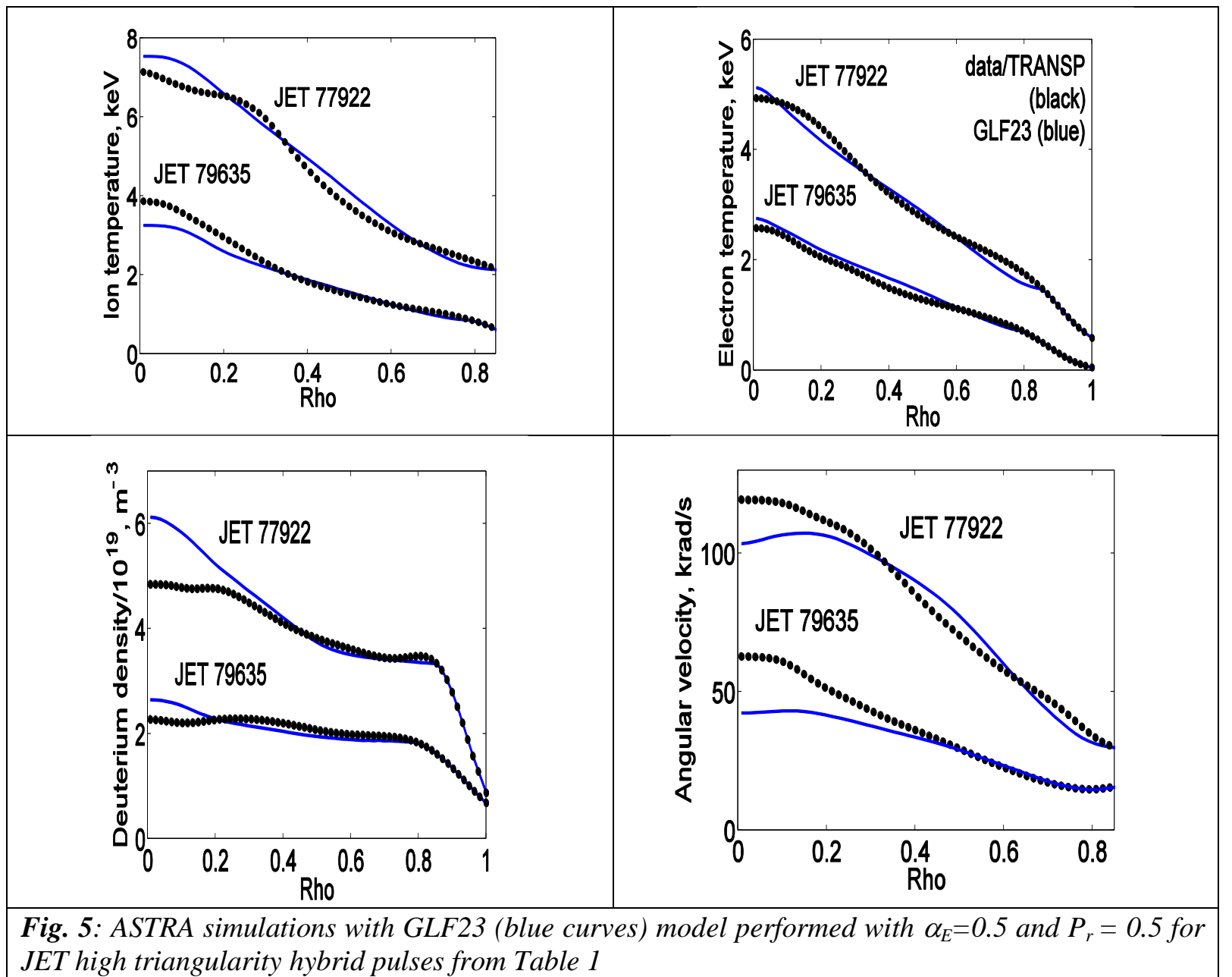

Similarly to the GLF23 model applied with the $\alpha_{E}=1$ as for the JET H-mode plasmas, an over-estimate of the electron density peaking by $15 \%$ in JET hybrid scenarios has been found in the self-consistent JETTO simulations of electron density and electron and ion temperatures performed with the H-mode version of the Bohm-gyroBohm transport model [33]. To simulate the measured density profiles in the high power $\left(\mathrm{P}_{\mathrm{NBI}}>17 \mathrm{MW}\right)$ hybrid pulses the core particle diffusivities have to be higher by a factor 1.5-2.0 with respect to the standard Bohm/gyro-Bohm transport model for both the low and high triangularity cases while assuming zero particle pinch (Bohm-gyroBohm predicts correctly the peaking of the density profile in JET H-mode plasmas with an inward particle pinch). On the other hand, the behaviour of the temperature and q profile is in general correctly predicted. The reasons for an increase core particle diffusivity in high power $\left(\mathrm{P}_{\mathrm{NBI}}>17 \mathrm{MW}\right)$ hybrid discharges are not clear and should deserve specific investigation.

Summarising the results of this section, the ExB shear stabilisation as included in the GLF23 model is found to be weaker in selected JET Hybrid scenario as compared to the H-mode plasmas and high $\beta_{N}$ scenarios. This conclusion is confirmed also by the CRONOS simulations of JET and ASDEX-Upgrade hybrid scenario shown in the previous section where a good agreement with measurements has been obtained by neglecting the ExB shear stabilisation. These results complement transport modelling of ASDEX-U discharges [25] where it is found that inclusion of ExB shear has little influence on reproducing the experimental temperature profiles in simulations using the Weiland model. For DIII-D, GLF23 simulations were performed with or without including the effect of ExB flow shear 
[34]. In the high rotation case, inclusion of the $E x B$ flow shear yields a significantly better match to the profiles. In the low rotation case, an equally good match is found either with or without the flow shear. Similar effect, i.e. larger influence of the ExB shear on turbulent transport at high rotation can be seen also on Fig. 4. Indeed, looking more closely at Fig. 4, one can see that the GLF23 predictive accuracy for $T_{i}$ is better for the low-medium rotation shots $(74641,74634,79635,77922)$ while $T_{i}$ is under-estimated in four other discharges with high rotation. This gives an indication that a higher $\alpha_{E}$ value needs to be applied in GLF23 model to get better temperature prediction at high rotation. However, our approach was to find the $\alpha_{E}$ parameter which could satisfactory described the whole dataset. The best match over the whole dataset has been obtained with $\alpha_{E}=0.5$.

The turbulence/anomalous transport quench point at relatively low $\alpha_{E}$ in Hybrid scenario can be partly understood by taking into account other stabilising effects, such as $s / q$ and $\beta_{e}$ effects on the ITG turbulence (the stabilising effect of $\beta_{e}$ for JET \#77922 has been found in the linear electromagnetic GYRO simulations). The stabilising $\beta$ effect has also been analysed in reference [35] using theory based model on JET beta scan experiments. In this reference it was also found that Shafranov shift parameter has a destabilizing effect on linear growth rates. The simulations of toroidal rotation in Hybrid scenario with the GLF23 model give clear indication of the toroidal momentum pinch $\left(P_{r}<1\right)$ in considered plasmas where the ITG mode is dominant. Both the GLF23 and Bohm-gyroBohm models consistently over-estimate of the density peaking in high power Hybrid scenario when their H-mode settings are applied [33]. The over-estimation of the density peaking should be taken into account when applying these models to the estimation of ITER hybrid performance.

\subsection{Modelling of hybrid termination}

The termination of the JET hybrid discharges with the transition from the hybrid performance to the type III ELMy H-mode with subsequent H-L transition to the ohmic plasma has been analysed, allowing determination of the back-transition conditions. The termination occurs by switching off the NBI heating during the plasma current plateau with the subsequent $I_{p}$ ramp down and reduction of the magnetic field in the ohmic phase. An example of such simulations performed for \#77922 is shown on Fig. 6. The JETTO simulations of electron density and electron and ion temperatures have been performed for the whole plasma region including pedestal by using the non-local H-mode Bohm-gyroBohm transport model completed with the continuous ELM model where the pedestal height is controlled by the ballooning stability limit with the normalised critical pressure. The edge boundary conditions are prescribed at the plasma separatrix. To simulate the measured density profiles we have used the same prescription as discussed in section 2.3: in the high power $\left(\mathrm{P}_{\mathrm{NBI}}>17 \mathrm{MW}\right)$ hybrid pulses the core particle diffusivities have to be higher by a factor 2.0 with respect to the standard Bohm/gyro-Bohm transport model for both the low and high triangularity cases while assuming zero particle pinch [33]. During the hybrid performance the thermal flux through the separatrix determined mainly by the large NBI heating exceeds the $1.5^{*} P_{L-H}$ (where the threshold power of the L-H transition $P_{L-H}$ is determined in [36]) maintaining the H-mode-like pedestal. When the NBI power has been switched off during the $I_{p}$ plateau, the observed transition from the type I to type III ELMy H-mode is predicted relatively accurately by reducing the L-H power threshold (from $1.5^{*} P_{L-H}$ to $P_{L-H}$ ) with simultaneous reduction of $\alpha_{c r}$ from 1.6 (during the type I ELM phase) to 0.6. The selected $\alpha_{c r}=1.6$ for the type-I ELMy Hmode phase is the standard JET value in agreement with edge ballooning MHD stability for carbon wall experiments. $\alpha_{c r}=0.6$ for the type-III ELMy H-mode phase was adjusted to fit the measured pedestal energy in the dithering phase. Finally, further transition from the type 
III ELMy H-mode to ohmic plasma with L-mode edge is performed in simulations by switching from the H-mode to the L-mode Bohm-gyroBohm transport model [37].

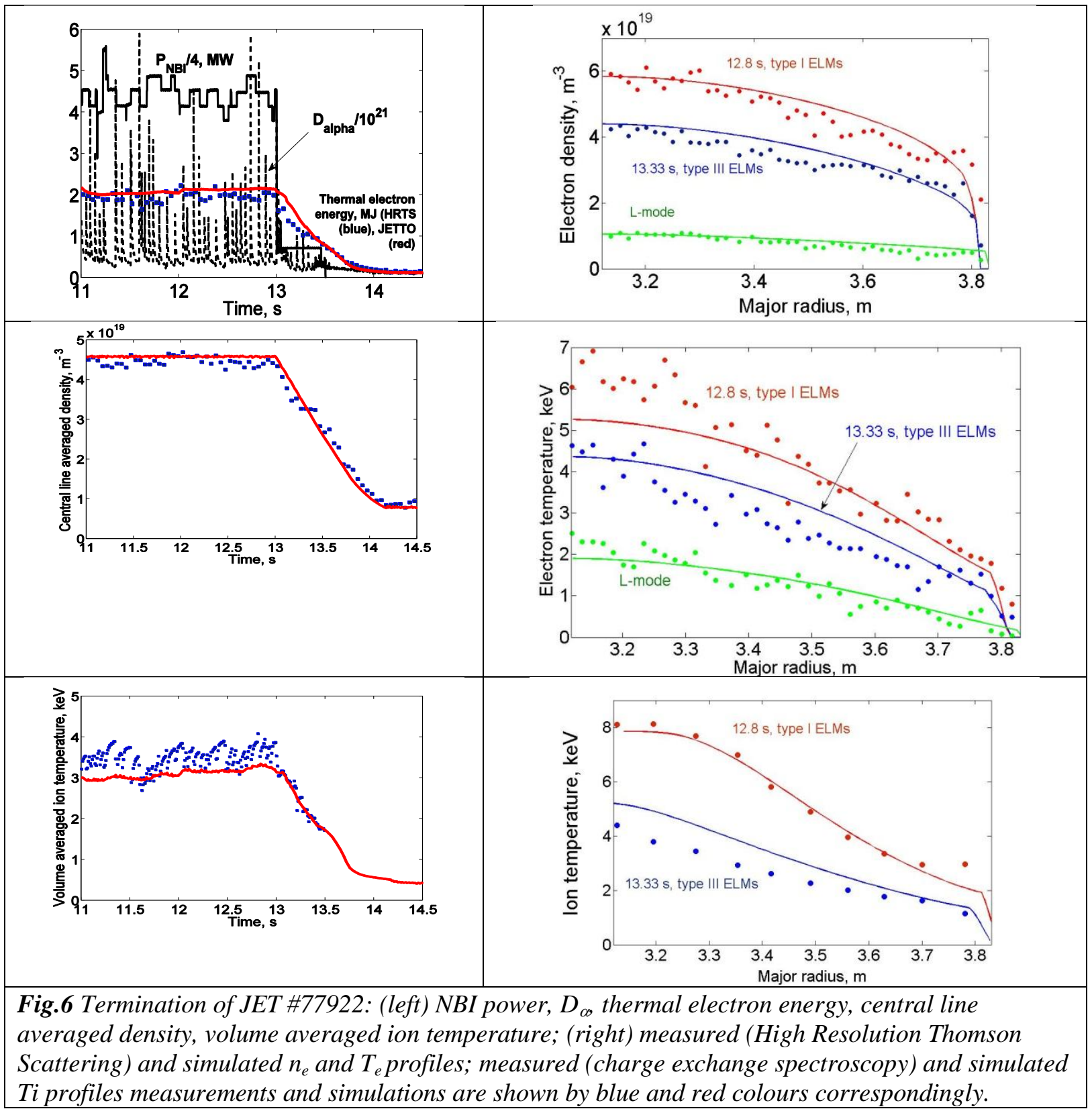

The L-mode Bohm-gyro-Bohm model has been used to carry out predictive simulations of the purely ohmic current ramp down phase of JET hybrid discharges, for different current rampdown rates $(0.17-0.21 \mathrm{MA} / \mathrm{s})$ and plasma densities at the beginning of the $I_{p}$ ramp down $\left(n_{l}=0.8-1.1 \times 10^{19} \mathrm{~m}^{-3}\right)$. Initial profiles and boundary conditions for plasma density, ion and electron temperatures and current density have been taken from the experimental signals, as well as the ramps in total plasma current and toroidal magnetic field, the effective ion charge, the radiated power and the gas puffing rate. The model has been used to self-consistently predict the time evolution of the electron density, ion and electron temperatures and current density profiles. A good match between the experimental and simulated time traces for the plasma internal inductance, the line-averaged electron density and the volume-averaged electron temperature is obtained provided that the particle recycling coefficient is increased typically from 0.5 to 1 during the ramp-down phase while the injected gas is reduced down to 
zero. A typical result of such an exercise on predictive modelling is shown in Fig. 7 for the ramp-down phase of JET hybrid discharge \#77922 (c.f. Table 1). The agreement between the predicted and measured time traces is good, reflecting in the averaged quantities the same level of agreement found for the electron density and temperature profiles.

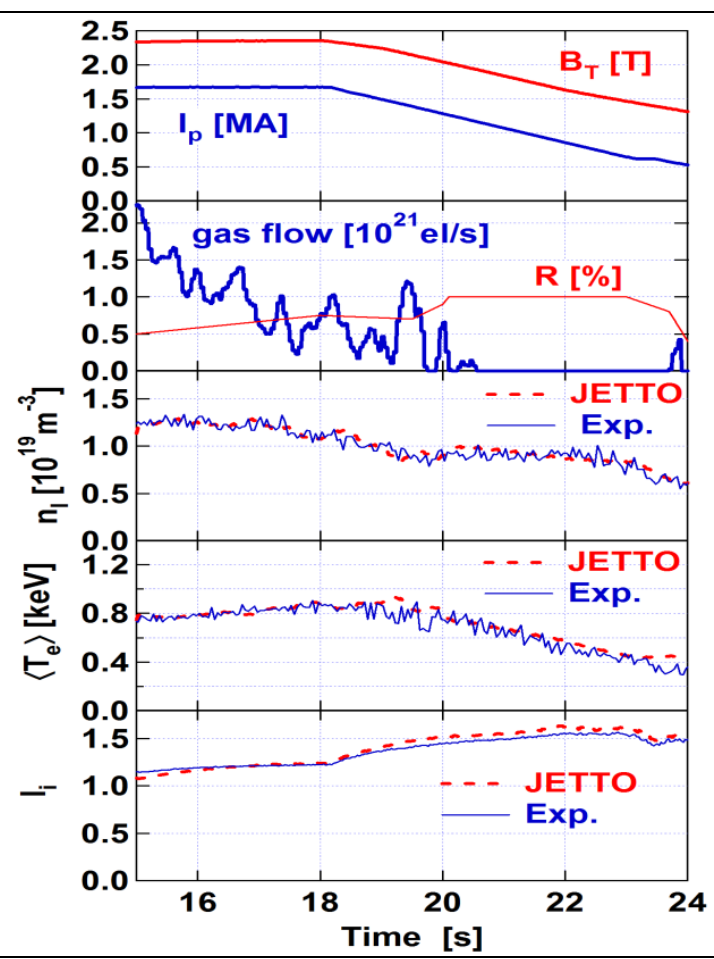

Fig. 7: Self-consistent current, temperature and density JETTO modelling of the JET hybrid discharge during the L-mode ramp down phase. 


\section{Predictive integrated modeling of ITER hybrid scenario}

Based on the understanding gained from modelling using a database of the present-day experiments, projections to ITER hybrid scenarios have been carried out focusing on optimization of the heating/current drive schemes to reach and ultimately control the desired q-profile shape with the ITER actuators and constraints.

\subsection{ITER hybrid operational domain from 0-D modeling}

A set of simulations of the ITER hybrid scenario is performed with the 0.5-D code METIS [38] which is a module included in the CRONOS suite of codes [9]. The main advantage of METIS consists in providing fast calculation in order to scan the operational domain and to define the domain where ITER hybrid scenario could exist while imposing the double constrain of having $q_{0}>1$ for long duration (1000s) and the ratio of fusion to additional powers, $Q_{D T}, Q_{D T}>5$.

METIS computes the time evolution of the global plasma quantities for given waveforms of the control parameters. It solves the current diffusion equation taking into account an approximate equilibrium evolution. Simplified treatment of the sources and of spatial dependences allow simulation of a discharge in a CPU time of the order of one minute, while keeping account of all the main non-linearities of the evolution. This approach allows completing the 0-D analysis with radial profiles and time evolutions, although with less accurate results than with a full 1.5-D code (which typically takes $10^{3}-10^{4}$ times larger computation times). As a result, these simulations can run in a CPU time which is close to real time and METIS is perfectly suited to test real time algorithm (c.f. section 3.5). METIS simulations for ITER have the following main characteristics:

1. a 2-D, time-dependent equilibrium is used, but based on equations for the time evolution of equilibrium moments: radii, elongation, triangularity, etc.,

2. heat transport coefficients are renormalized in order to enforce prescribed confinement scaling laws (in particular, L and $\mathrm{H}$-mode in the various phases of the discharge)

3. the full current diffusion equation is solved numerically

4. density and temperature profiles are obtained by simplified solutions of the transport equations: discrete time slices are considered, on which stationary equations are solved.

ITER hybrid scenarios have been calculated at a plasma current $I p=12 M A$ at $B_{T}=5.3 T$ $\left(q_{95}=4.3\right)$, with the ITER baseline heating mix 20MW ICRH, 33MW NBI, 20MW ECCD and with a line averaged density fixed to $n_{l}=7.5 \times 10^{19} \mathrm{~m}^{-3}\left(n_{l} / n_{G w} \sim 0.8\right)$ during the burn phase. The parameters that have been scanned in this sensitivity study are the density peaking factor to simulate flat and peaked density profiles with $n_{e o} / n_{l}=1,1.2,1.4$ and the enhanced confinement factor during the $\mathrm{H}$-mode phase from $H_{98 I P B(y, 2)} \sim 1.1,1.2,1.25,1.3,1.4$ with the corresponding pedestal pressure of $87 \mathrm{kPa}, 90 \mathrm{kPa}, 92 \mathrm{kPa}, 95 \mathrm{kPa}, 100 \mathrm{kPa}$ as deduced from a 0 -D scaling laws [39]. Fig. 8 (left) shows the time evolution of the plasma scenario for the high confinement case $\left(H_{98 I P B(y, 2)} \sim 1.4\right)$ with three different values of density peaking keeping the same line averaged density or Greenwald fraction (i.e. an increase of the density peaking is obtained by increasing the core density while reducing the pedestal one). With the assumed baseline heating mix and the neo-classical current diffusion, METIS calculations indicate that high confinement and peaked density profiles are required to increase the bootstrap current at level above a certain threshold ( $I_{\text {boot }} \sim 4 M A$ or $I_{b o o t} / I_{p} \sim 30 \%$ for the case shown on Fig. 8) to 
self-sustain the q-profiles above unity. Fig. 8 (right) presents the results of the full sensitivity studies where the bootstrap current fraction and the ratio of fusion to additional powers, $Q_{D T}$, have been plotted versus the enhanced confinement factor for the three density peaking. It confirms that the operational domain for the hybrid regime with $q_{0}>1$ for more than $1000 \mathrm{~s}$ and $Q_{D T}$ above 5 is relatively narrow and requires high confinement and peaked density profile to reach a critical value of bootsrap current fraction. These conclusions deduced from METIS are consistent with the recently proposed criterion to distinguish the different plasma regime: it was indeed found that a critical profile of bootstrap current characterises the hybrid regime [40]. It was shown that a critical value of bootstrap current is a condition for the transition from inductive H-mode to purely non-inductive regime and the hybrid scenario appears as an intermediate plasma state between these two states.

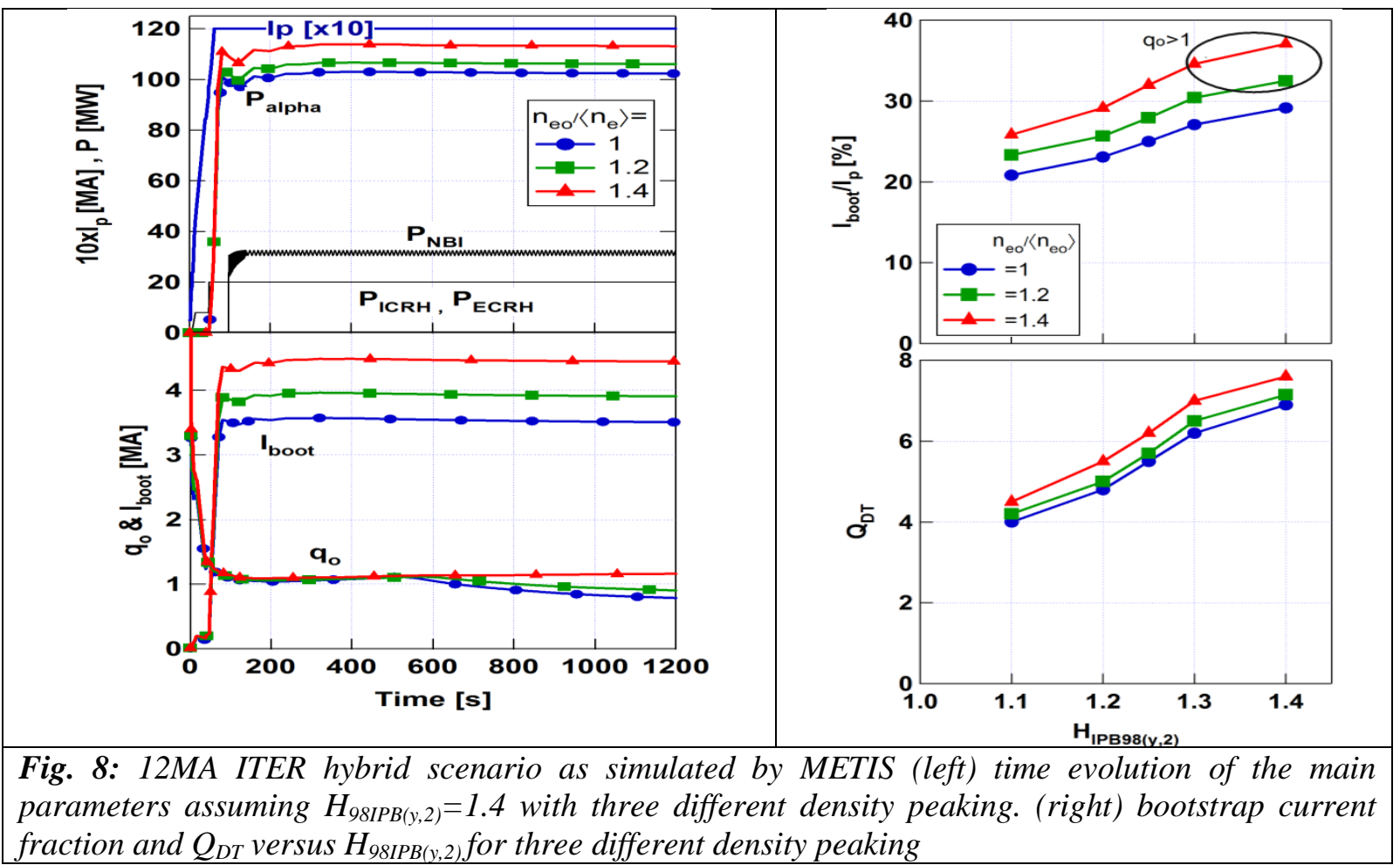

\subsection{Current profile optimization during current ramp-up phase}

Access condition to the class of hybrid-like q-profiles (i.e. flat in the core and slightly above unity with a high magnetic shear in the gradient zone) during the prelude phase of the scenario is investigated with particular attention in this section [41-43]. The plasma current ramp-up phase is a critical phase of the scenario preparation where the optimised q-profile should be reached while deleterious MHD instabilities for confinement and stability have to be avoided, flux consumption has to be minimized, and this has to be achieved within ITER operational constrain. Validation on the ramp-up phase of JET, ASDEX-Upgrade and Tore Supra [44-45] has shown that both empirical scaling based models and the semi-empirical Bohm/gyro-Bohm model (L-mode version) yield a good reproduction of this phase for considered discharges, in terms of $T_{e}$ and q-profile, i.e. $l_{i}$. Therefore these models have been used in the reported work, which was carried out with the CRONOS integrated suite of codes.

The optimisation of the current ramp-up phase (plasma current waveform, external heating \& current drive waveform, timing for $\mathrm{L}$ to $\mathrm{H}$ transition) is systematically investigated in view of (i) optimising the q-profile at the start of the current plateau for improved fusion performance, 
and, (ii) minimizing the resistive flux consumption to allow for long pulse operation while keeping the current in the central solenoid (CS) and poloidal field (PF) coils within the ITER operational limits. The optimisation of the q-profile relies on reaching a target q-profile that improves stability ( $\mathrm{q}$ above unity and weak shear in the core) and energy confinement (high magnetic shear in the gradient zone). In regimes with stiff profiles in the Ion Temperature Gradient dominated turbulent plasma, $R / L_{T i}$ stays in the vicinity of its threshold value, $R / L_{T i, c r i t}$ for triggering the micro-instabilities. Indeed, theories predict and it was confirmed in our predictive modelling of exiting experiments (section 2.2) that $R / L_{T i, \text { crit }}$ increases linearly with $s / q$. As a consequence, a figure of merit, $F$, for optimising in a quantitative manner the qprofile is defined as $F=\langle s / q\rangle \mathrm{q}_{\mathrm{a}}$ where $\langle s / q\rangle$ is the volume averaged values of $s / q$ [43]. A high value of $\langle s / q\rangle$ is achieved by $q_{0}$ close to 1 , a wide low shear region, and high $s$ in the outer part of the plasma, which are indeed the characteristics of a hybrid q profile. In the extreme case, the maximisation of $\langle s / q\rangle$ is done by the so-called current ramp overshoot as developed on JET. The minimisation of the resistive flux consumption is done by calculating the Ejima coefficients [46], $C_{E}$, at the end of the current ramp-up phase. The Ejima coefficient is the normalised resistive flux consumption to the poloidal flux. In the extreme case, the minimisation of $C_{E}$ is achieved by either early heating and/or early H-mode transition.

For the reference current ramp up modelling, the assumptions are as follow:

(i) Simulations start $1.5 \mathrm{~s}$ after breakdown, when $I_{p}=0.5 M A$. Current flat top $(12 \mathrm{MA})$ is reached at $80 \mathrm{~s}$ with an expanding elongated shape, starting on the Low Field Side of the torus (X-point formation at $15 \mathrm{~s}$, when $I_{p}=3.5 M A$ ).

(ii) The parabolic density profile with a peaking factor $n_{e d} /\left\langle n_{e}\right\rangle=1.3$ is increased with the prescription $n_{e}=0.25 x n_{e G w}$

(iii) A flat $Z_{\text {eff }}$ profile, decreasing in time from 5 to 1.7 with increasing density.

(iv) An L-mode edge during the whole ramp-up phase with applied power (after 50s) below the L-H power threshold ( 29MW).

During the ramp-up phase the plasma is heated with a combination of NBI using the off-axis setting, ECRH (Upper Port Launcher) and LHCD systems (Fig. 9). This combination of applied power offers the possibility to achieve a broad off-axis non-inductive current density profile to reach the required q-profile for the hybrid scenario. Fig. 10 shows the electron, ion and q-profiles for the reference case with two transport assumptions: empirical scaling based model with $H_{I P B 98(y, 2)}=0.4$ and the L mode Bohm/gyro-Bohm model. The ohmic reference case is also shown for comparison.

Around this reference case, various ITER current ramp-up scenarios have been modelled, i.e. (i) with and without plasma current overshoot, (ii) with early heating, (iii) with or without early H-mode transition. A summary of the studies is shown on Fig. 11 where the Ejima coefficient, $C_{E}$, and the figure of merit, $F$, for the q-profile optimisation are plotted versus the total input energy during the ramp-up phase, $W_{\text {input }}$. It is concluded that a trade-off should be found between minimising the resistive flux consumption and optimizing the q profile. A trade-off between these two requirements has to be made. It is shown that fast current ramp with current overshoot is at the one extreme, i.e. optimum q profile at the cost of increased resistive flux consumption, whereas early $\mathrm{H}$-mode transition is at the other extreme. It is also found that the heating systems available at ITER allow, within the operational limits, to reach a hybrid q-profile at the end of the current ramp-up. The optimum heating scheme depends on the chosen transport model. Nevertheless, modified model assumptions (on density peaking, edge temperature and effective charge) can be easily accounted for by a tuning of the power waveform during the prelude phase. The flexibility of the heating system open the route to an 
active control of the q-profile during the ramp-up phase to reach the required target values as it will be further discussed in section 3.5.

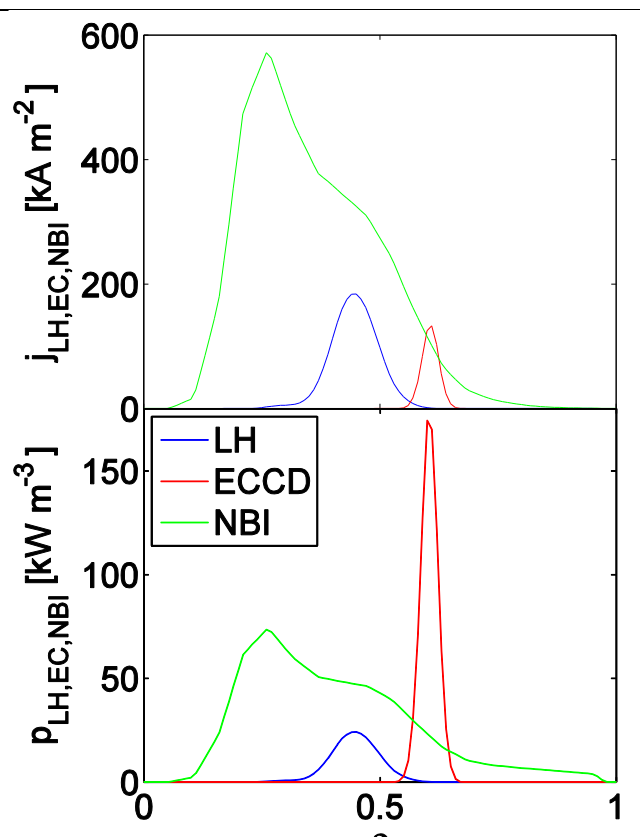

Fig. 9: Simulated driven current (top) and absorbed power (bottom) density profiles, plotted versus normalized toroidal flux coordinate $\rho$ at the end of the current ramp-up $80 \mathrm{~s}: 8 \mathrm{MW}$ of ECCD from one of the UPL antennas (red), 3MW of LHCD (blue) and 16.5MW of NBI (green).

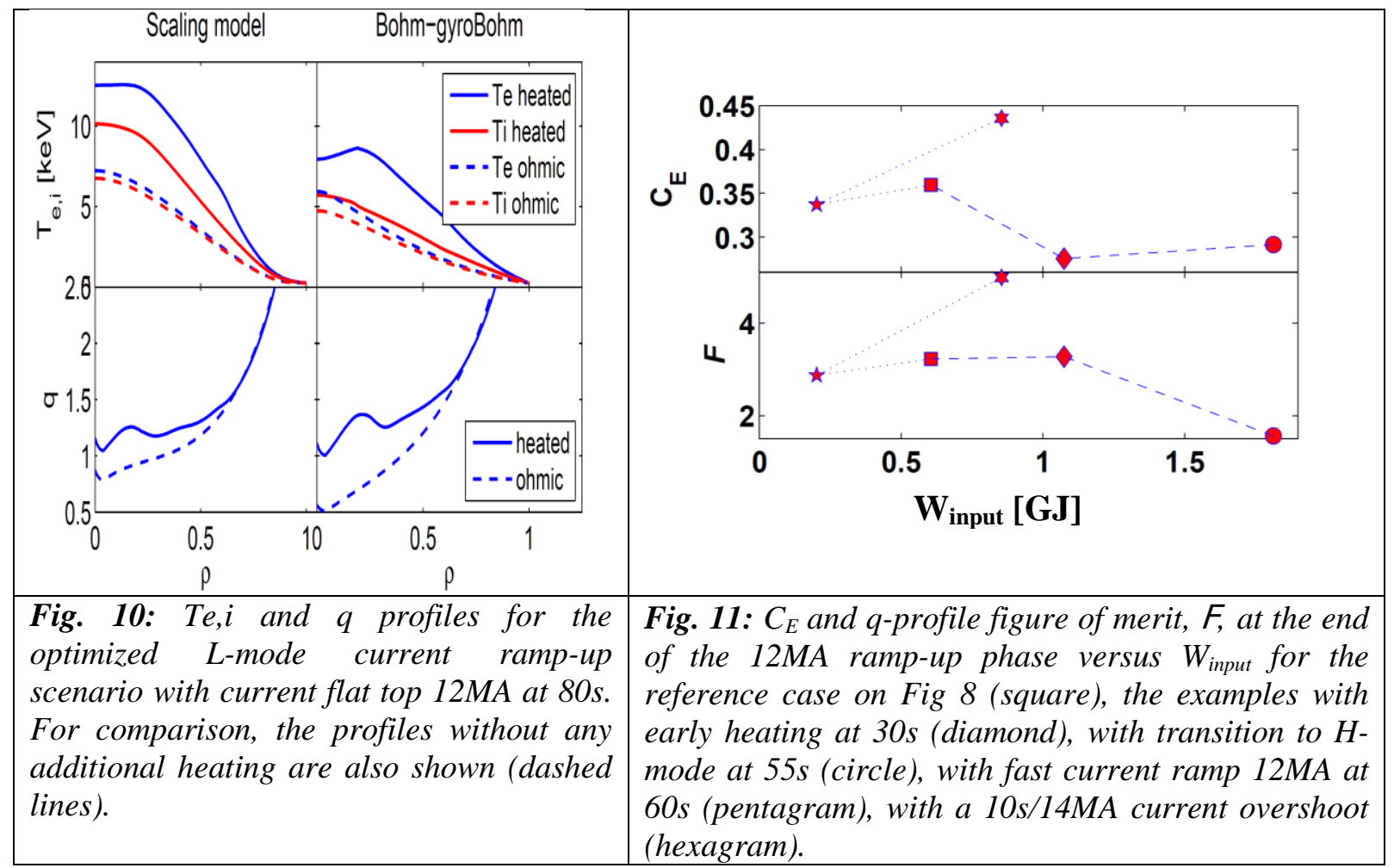




\subsection{Pedestal prediction with first principle predictive model}

Accurate prediction of the edge transport barriers is essential to assess and optimise ITER fusion performance. In this context, the EPED pedestal model [47-51] has been applied to ITER hybrid scenarios. EPED is a first-principle model for predicting the $\mathrm{H}$-mode pedestal height and width based upon two fundamental and calculable constraints: (1) onset of nonlocal peeling-ballooning modes at low to intermediate mode number, (2) onset of nearly local kinetic ballooning modes at high mode number. Indeed, the peeling-ballooning stability limit provides a global constraint on the pedestal height as a function of the pedestal width. The kinetic ballooning stability limit provides the mechanism by which the pressure gradient is finally constrained. Calculation of these two constraints allows a unique, predictive determination of both pedestal height and width without any free or fitting parameters. The EPED model has been extensively tested across a range of experiments on several devices on a large database of 5 tokamaks (JET, DIII-D, JT-60U, Alcator C-Mod, ASDEX-Upgrade) consisting of more than 250 entries. The EPED model was found to be in good agreement with the observations, with a ratio of predicted to observed pedestal height of $0.98 \pm 0.2$ [48]. More recently, the EPED model has been validated on the specific JET hybrid database (77 cases) where experimental scan in plasma shaping (triangularity), pedestal density and global beta has been provided. It was found that the variation of pedestal height with respect to the pedestal density, triangularity and global beta was correctly captured by the proposed model with a ratio of predicted to observed pedestal height of $0.89 \pm 0.12$ [50].

As a practical consequence, the EPED pedestal model has been applied to ITER hybrid scenarios. The inputs to the model are nine scalar parameters: $B_{t}(T), I_{p}(M A), R(m), a(m), \delta, \kappa$, $n_{e, p e d}\left(10^{19} \mathrm{~m}^{-3}\right), Z_{e f f}, \beta_{N}$, where $n_{e, p e d}$ is the pedestal electron density. For the ITER hybrid simulation the following equilibrium parameters were set to $R=6.2 \mathrm{~m}, a=2 \mathrm{~m}, \kappa=1.85, \delta=0.485$, $B_{t}=5.3 \mathrm{~T}$. Predictions for the hybrid scenario have been made for the pedestal height and width at various plasma currents $\left(I_{p}=11,12,13 \mathrm{MA}\right)$, effective charge $\left(Z_{\text {eff }}=1.7,2.5\right)$, pedestal density $\left(n_{e, p e d}=6.5,7.5,8.5,9.5,10.5 \times 10^{+19} \mathrm{~m}^{-3}\right)$ and $\beta_{N}=1.8,2.2,2.6,3.0$. For this density range, it was found that the $\beta_{N}$ dependence is weak and the results shown on figure 12 have been obtained for $\beta_{N}=2.2$. One key feature of the hybrid scenario is the operation at reduced plasma current for reaching long pulse operation. The interaction of the peeling-ballooning and kinetic ballooning constrains predicts that the pedestal height and width changes with the plasma current. The results of the $I_{p}$-scan are shown on figure 12 (left) where the pedestal heights are plotted versus the pedestal width for various densities and for two $Z_{\text {eff }}$ values (at $\beta_{N}=2.2$ ). The global peeling-ballooning stability limit increases roughly linearly with $I_{p}$ whereas the kinetic ballooning stability limit increases with $I^{2}$. The combination of the two MHD limits leads to a pedestal height that first rises and then stagnates while the pedestal width decreases with $I_{p}$. Furthermore, Fig. 12 shows that by increasing $Z_{\text {eff }}$ from 1.7 to 2.5 increases the predicted pedestal pressure. Similarly, EPED model predicts that the pedestal height increases with density for the analysed density range (i.e. density below Greenwald density limit ) (Fig. 12 (right)). These two dependences (with $Z_{\text {eff }}$ and density) are interpreted through the collisionality dependence of the kink/peeling stability limit. 

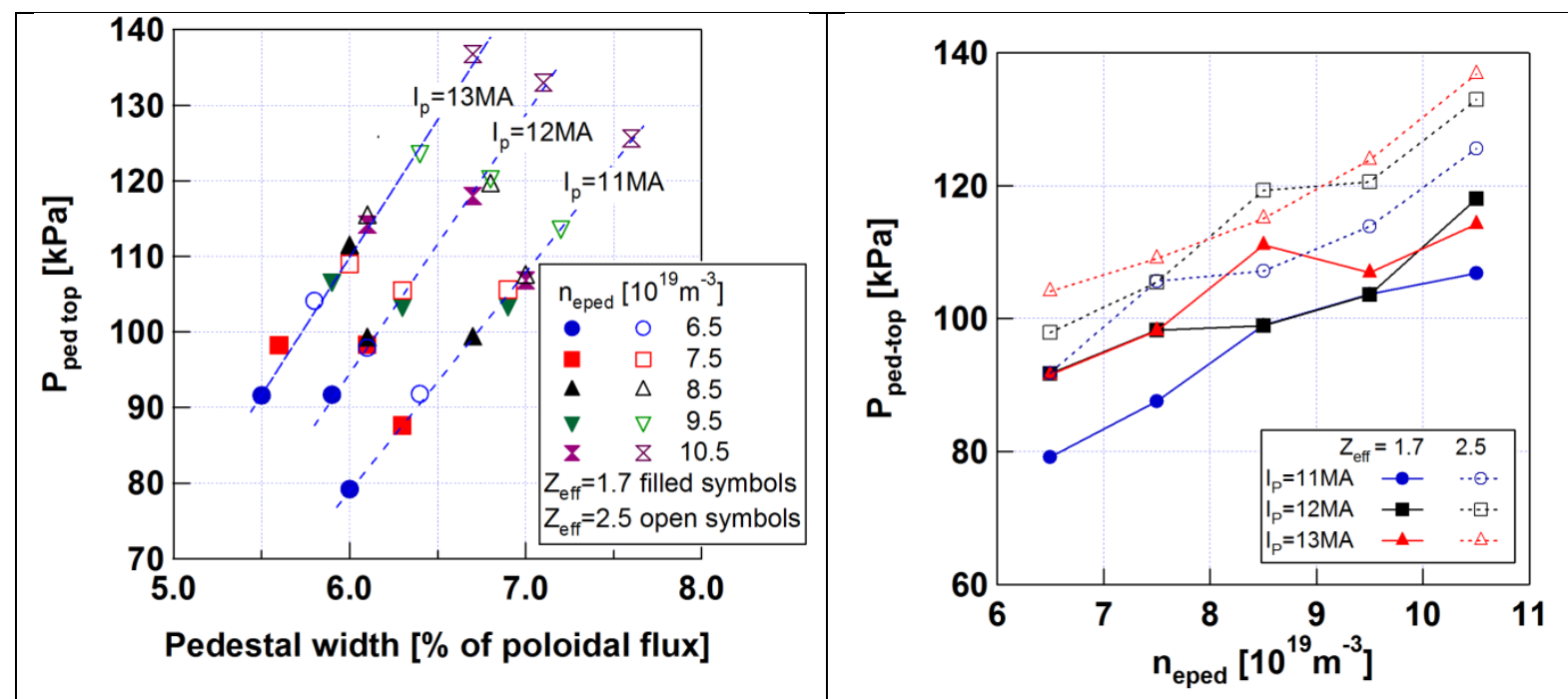

Fig. 12: EPED prediction of the pedestal width and height for the range of parameters of the ITER hybrid scenario (left)Pressure at the top of pedestal versus pedestal width for different pedestal densities, $n_{\text {eped }}, Z_{\text {eff }}$ and plasma current. (right) Pressure at the top of pedestal versus pedestal density for different plasma current and $Z_{\text {eff }}$ values.

\subsection{Consistent core and pedestal integrated modeling}

In this section hybrid scenario performance in ITER is studied with the CRONOS integrated modelling suite, using the GLF23 anomalous transport model for heat transport prediction. The modelling is performed by imposing the values for the pedestal width and height as calculated separately by EPED. From the interpreted role of the $s / q$ ratio in experiments, ITER hybrid scenario flat-top performance has been optimized through tailoring the q-profile shape, for various assumed pedestal conditions [52]. The optimum q-profile shape is predicted to be one that maximizes the ratio of $s / q$ throughout the bulk of the plasma volume [27]. In the proposed study, we investigate the importance of the density peaking on the fusion performance and q-profiles using simultaneously first principle models for the core heat transport and pedestal width.

The scenario is an extension to the one published in [52] since the pedestal parameters are obtained from EPED model. ITER hybrid scenarios were calculated at a plasma currents $I p=11.5-11.8 M A$ (depending on the precise case) at $B_{T}=5.3 T(\mathrm{R}=6.2 \mathrm{~m}, \mathrm{a}=2 \mathrm{~m}, \kappa=1.89$, upper triangularity $\delta_{\text {upper }}=0.454$, lower triangularity $\delta_{\text {lower }}=0.516$ ) with the ITER baseline heating mix 33MW NBI (1MeV, full off-axis injection), 20MW ECRH (equatorial launchers, angles varied between $20^{\circ}$ and $45^{\circ}$ ), 20MW ICRH (53 MHz, 2nd $\mathrm{T}$ harmonic), and with a line averaged density fixed to $n_{l}=8.8 \times 10^{19} \mathrm{~m}^{-3}\left(n_{l} / n_{G w} \sim 0.95\right)$ during the burn phase. The main CRONOS assumptions are as follows: equal ratios of $\mathrm{D}$ and $\mathrm{T}$ are assumed, the $\mathrm{Z}_{\mathrm{eff}}$ profile is flat with a fixed value of 1.67, q-profile evolution is predicted by modelling the current diffusion with the neoclassical resistivity calculated by the NCLASS model, electron and ion heat transport are predicted, the density profile is prescribed. Rotation is set to zero (a conservative assumption) and GLF23 is applied with $\alpha$-stabilization off. GLF23 calculates the anomalous transport in the core for the bulk of the volume inside the pedestal top, between $\rho=0.25-0.92$. For $\rho<0.25$ a constant $\chi_{\mathrm{e}, \mathrm{i}}=0.5 \mathrm{~m}^{2} \mathrm{~s}^{-1}$ is assumed, due to the GLF23 predicted stability in that region.

As in the METIS calculation discussed in section 3.1, three different values of density peaking $n_{e d} / n_{l}=1,1.25,1.5$ have been selected while keeping the same line averaged density or 
Greenwald fraction (i.e. an increase of the density peaking is obtained by increasing the core density while reducing the pedestal one). It is worth mentioning that the 1.5 peaking factor is predicted by collisionality scaling deduced from experiments [53]. For the sake of simplicity linear $\mathrm{n}_{\mathrm{e}}$ profile shapes are assumed. The temperature pedestal tops are set at $\rho=0.92$ in accordance with the EPED predicted pressure height and widths while assuming equal electron and ion temperature. These predicted values differ for each of the prescribed pedestal top density values corresponding to the three assumed density peaking factors. The pedestal top $\left(\Delta_{\text {top }}\right)$ is defined as the distance (in units of normalized poloidal flux $\psi$ ) corresponding to $\mathrm{x} 1.5$ the 'pedestal width' $\Delta$ which parameterizes the hyperbolic tangent kinetic profile shapes defined in equation 1 in [47]. In our simulations, this location sets the boundary values for the GLF23 predictions. The CRONOS produced pedestal profiles are not themselves hyperpolic tangent shapes, but the temperature pedestal heights and widths were adjusted such that the smooth transition to the GLF23 predicted transport coefficients occurs at the EPED predicted $\Delta_{\text {top }}$, at EPED consistent pedestal top pressures.

The results of the simulations are summarised in Table 2 while the kinetic and q-profiles produced at the end of the burn phase (1200s) are shown on Fig. 13. When imposing first principle calculation for the core and pedestal transport and with the ITER baseline heating and current drive mix, the calculation indicate that: (i) the thermal enhanced confinement factor, $\mathrm{H}_{\mathrm{IPB} 98(\mathrm{y}, 2)}$ is around unity, (ii) the resulting bootstrap current fraction is around $30 \%\left(\beta_{N}\right.$ 2) which is the marginal value to maintain the q-profile above unity as also suggested by the METIS simulation in section 3.1, (iii) the increase of the density peaking at fixed density weakly affects the fusion performance and the ability to sustain $\mathrm{q}_{0}$ above unity for more than 1000s. Indeed, when increasing the density peaking, the density at the pedestal top is reduced which leads in these consistent core-pedestal calculations with EPED to a reduction of the pressure at the pedestal top as discussed in section 3.3. In this case, the increase of core fusion performance and core bootstrap current due to the increase of core density are counter balanced by the reduction of the pedestal confinement properties and bootstrap current in the pedestal region. It is worth noting that in all these scenarios, replacing the ICRH with increased off-axis ECCD (beyond the ITER baseline provision) at $\rho \sim 0.35$ improves the scenario by providing increased off-axis non-inductive current significantly reducing the volume of $q<1$ while maintaining optimum q-profile shaping as expressed by the $\langle s / q\rangle$ parameter of merit. 


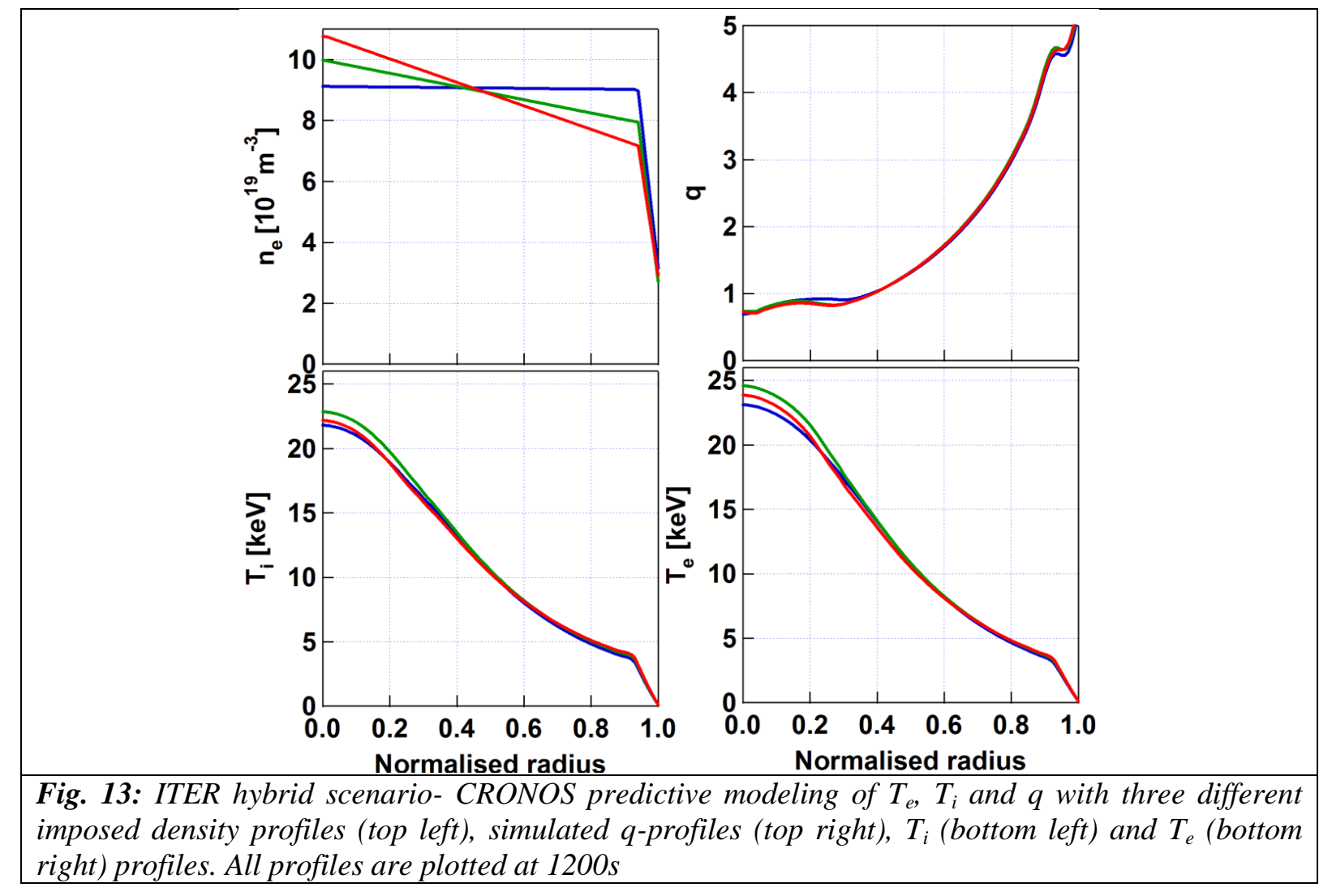

Table 2: Summary of results for CRONOS modelling of ITER hybrid scenario with GLF23 and pedestal parameters calculated with EPED for three density peaking. All evolving parameters are quoted at 1200 s.

\begin{tabular}{|l|l|l|l|l|l|l|l|l|}
\hline$n_{e d} / n_{l}$ & $\begin{array}{l}n_{\text {e,top }} \\
{\left[10^{19} \mathrm{~m}^{-3}\right]}\end{array}$ & $\begin{array}{l}T_{i, \text { top }} \\
{[\mathrm{keV}]}\end{array}$ & $\begin{array}{l}P_{\text {top }} \\
{[\mathrm{kPa}]}\end{array}$ & $\Delta_{\text {top }}\left[\psi_{\text {norm }}\right]$ & $Q$ & $I_{\text {boo }} / I_{P}$ & $\beta_{N}$ & $H_{\text {IPB98(y,2) }}$ \\
\hline 1 & 9.02 & 3.67 & 96.3 & 0.064 & 4.71 & $30 \%$ & 1.91 & 1.06 \\
\hline 1.25 & 7.99 & 3.9 & 90.2 & 0.064 & 5.06 & $33 \%$ & 1.97 & 1.08 \\
\hline 1.5 & 7.24 & 4.02 & 84.4 & 0.064 & 5.06 & $33 \%$ & 1.93 & 1.05 \\
\hline
\end{tabular}

\subsection{Model-based magnetic and kinetic real time control}

In hybrid or steady state scenarios, simultaneous magnetic and kinetic control of plasma profiles and parameters such as the current profile, the pressure profile (or the normalized pressure parameter, $\beta_{N}$ ), and the alpha-particle power are essential to maintain high performance for durations that exceed the resistive diffusion time. An integrated model-based plasma control strategy, ARTAEMIS, has been initiated on JET [54] and pursued on JT-60U and DIII-D [55], and closed-loop control of the poloidal flux, safety factor and $\beta_{N}$ has been recently performed [56].

The general model-based approach has also been applied to the ITER hybrid regime for the control of the magnetic equilibrium (poloidal flux profile) and of the alpha-particle power, $P_{\alpha}$ using six actuators [57]. The control actuators are the two ITER neutral beam injectors (NBI1, NBI2), electron cyclotron (ECRH), ion cyclotron (ICRH) and lower hybrid (LHCD) systems, and the plasma surface loop voltage $\left(V_{e x t}\right)$. In practice, the surface loop voltage is obtained from the tokamak plasma control system through a separate control system that uses the ohmic coil voltage actuator. This separate control is not modeled here. The central poloidal 
field coil is therefore used for controlling $V_{\text {ext }}$ rather than for controlling the total plasma current, $I_{p}$, and $I_{p}$ is controlled indirectly through the controlled magnetic profiles. The nonlinear plasma response to the actuators is modeled through the time evolutive METIS transport code. The controlled parameters are the poloidal flux profile $\Psi(x, t)$, and $P_{\alpha}$. Based on the simulated response data obtained from METIS to modulations from the six actuators, a full, two-time-scale model was identified using the ARTAEMIS algorithm. Then this model was validated on different METIS simulation data. Closed-loop control simulations were performed by inserting the METIS code at the output of the two-time-scale ARTAEMIS controller and feeding the appropriate error signals back into the controller, thus closing the loop. The near-optimal controller design parameters [54] were computed using the identified model, and the various weights in the controller cost function and in the steady state objective were adequately tuned. The evolutions of the $P_{\alpha}$ and poloidal flux profile $\Psi(x, t)$ in closedloop control simulation are shown in Fig. 14 together with the reset references values. The trajectory to reach the reference equilibrium is a consequence of the optimal control where the time to reach the target profiles is minimized together with the cost of the control action in terms of actuator power. In these simulations, various target profiles for the poloidal flux have been obtained simultaneously with various target levels of fusion power. The modelling indicates that in a fusion device such as ITER, magnetic poloidal profile control can be combined with burn control, sharing a common set of dedicated actuators. The choice of the controlled variables that was made here, namely the poloidal flux profile and the alphaparticle fusion power, was the simplest one for the first proof of principle tests of a two-timescale state-feedback controller. However, the safety factor profile, $q(x)$, which is closely linked to the poloidal flux profile, is an important physical quantity that governs stability and confinement in tokamak plasmas. In the future, this work will be extended to control not necessarily the poloidal flux profile but rather the safety factor profile. It is of course more demanding in terms of modeling, real-time measurements and control because it depends on the radial derivative of the poloidal flux. Preliminary experiments were carried out on DIII-D where optimal feedback control [56] of the safety factor profile through its inverse $(1 / \mathrm{q}(\mathrm{x}))$ was attempted during the ramp-up phase. In a near future, this work will be extended for application to the modeling of the profile control of ITER scenario.

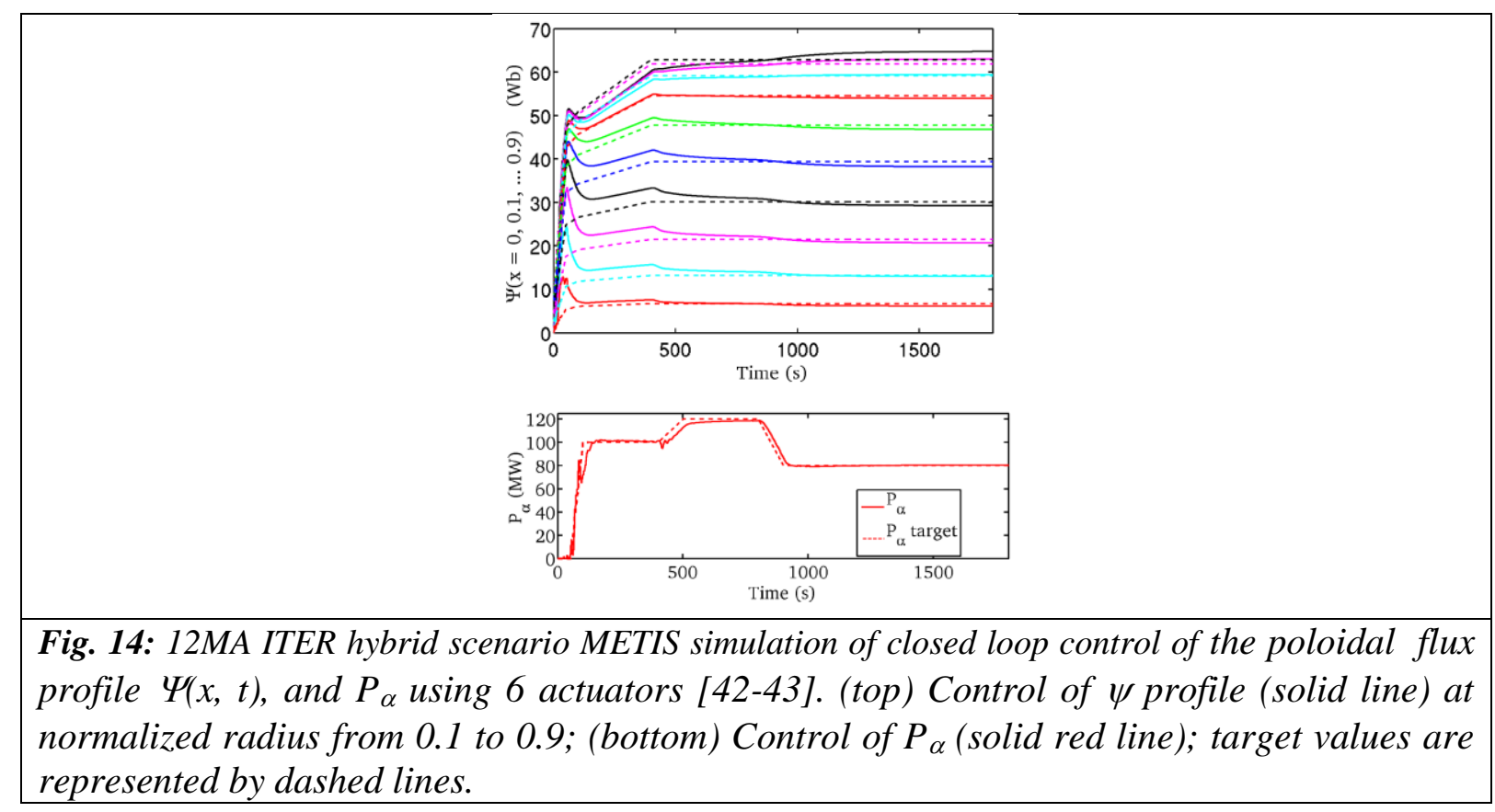




\section{Discussion and Conclusion}

An extensive analysis of hybrid scenarios on JET and ASDEX-Upgrade including current diffusion, global energy confinement, core and pedestal transport, pedestal stability and H-L transition, has been performed by the ISM working group in 2010-2012 and is summarised as follows:

- Current diffusion is in agreement with the neoclassical prediction for JET discharges while discrepancy with the MSE data has been found for ASDEX-Upgrade, with a much faster inward current diffusion obtained in simulations as compared to measurements.

- Observed improved confinement in hybrid scenarios is partly explained by the qprofile modification maximising $s / q$ ratio in the outer part of the plasma region which accounts for $\sim 60-90 \%$ and $\sim 35-55 \%$ of the observed $\sim 20 \%$ confinement improvement in JET and ASDEX-Upgrade correspondingly [27]. Linear electromagnetic GYRO simulations of the high $\beta_{N}$ JET hybrid discharge \#77922 show a strong reduction of the ITG growth rate with $\beta_{e}$ indicating that finite $\beta$ effect can be another stabilising factor for anomalous transport. In contrast, the $E x B$ shear stabilisation as included in the GLF23 model is found to be weaker in selected JET hybrid discharges as compared to the H-mode plasmas and high $\beta_{N}$ scenarios. The reduction of turbulence/anomalous transport quench point (i.e. low $\alpha_{E}$ ) in hybrid scenario can be partly understood by taking into account other stabilising effects on the ITG turbulence, such as $s / q$ and $\beta_{e}$.

- An extensive validation of the GLF23 and Bohm-gyroBohm transport models during the main heating phase of hybrid scenarios performed in the self-consistent manner (up to four-field density, temperatures and momentum) indicates that:

a. Simulated density profile is over-peaked both in simulations with the Bohm-gyroBohm models applied with the H-mode settings and GLF23 model applied with the $\alpha_{E}=1$ typically used for the JET H-mode and high $\beta_{N}$ plasmas. The re-tuning of the Bohm-gyroBohm model (reduction of particle diffusion by factor 2 and neglecting the particle pinch) has been proposed [33]. In simulations with the GLF23 model a good agreement with measured density is achieved by reducing $\alpha_{E}$ by factor 2 . These results should be taken into account when applying the GLF23 and BohmgyroBohm models to the estimation of ITER hybrid density profile.

b. Electron and ion temperature are reasonably well predicted with both GLF23 and Bohm-gyroBohm models.

c. Simulations of toroidal rotation have been performed for the first time for the JET hybrid scenarios with the current overshoot by using the GLF23 [30]. Using the GLF23 computed momentum transport strong overprediction of toroidal rotation velocity has been obtained. A relatively good agreement with measured toroidal velocity has been achieved when applying the fraction of the GLF23 computed thermal ion diffusivity for momentum transport. The Prandtl number found in these simulations is $P_{r}=0.3$ and $P_{r}=0.5$ in low and high triangularity discharges correspondingly. 
- The EPED model prescribing the width and height of pedestal pressure has been validated on a number of JET hybrid scenarios performed in broad range of pedestal densities and global beta, and different triangularities showing a good agreement with the measurements [50].

- Termination of JET hybrid scenarios including the H-L transition at the end of the NBI heating with the subsequent plasma current and magnetic field ramp down phase has been simulated including the density, $T_{e}$ and $T_{i}$ evolution. The transition from hybrid performance with type I ELMs to type III ELMy H-mode has been reproduced in simulations with the Bohm-gyroBohm transport model and continuous ELM model by reducing the ballooning stability limit and L-H threshold power by $40 \%$. Subsequent transition from type III H-mode to ohmic plasma has been performed with the reduction of power below the $\mathrm{L}-\mathrm{H}$ threshold by switching from the $\mathrm{H}$-mode to Lmode Bohm-gyroBohm model. It has been found also that the Bohm-gyroBohm model accurately predicts the temperatures and density evolution during the current ramp down phase.

The transport and pedestal stability models validated on existing hybrid scenarios (GLF23, Bohm-gyroBohm and scaling-based thermal transport models, EPED) have been applied in the modelling of ITER hybrid scenario. The GLF23 model has been used without the ExB shear stabilisation since the effect of the $\operatorname{ExB}$ shear is found to be weak in JET and ASDEXUpgrade hybrid discharges. The EPED model has been extrapolated to ITER by performing the pedestal simulations within a broad range of ITER hybrid parameter space used for scenario optimisation $\left(I_{p}=11-13 \mathrm{MA}, Z_{e f f}=1.7\right.$ and $2.5, n_{e, p e d}=6.5-10.5 \times 10^{19} \mathrm{~m}^{-3}$ and $\left.\beta_{N}=1.8-3\right)$. The objective of these simulations was the optimisation of ITER hybrid performance by taking advantages of the $s / q$ stabilisation of the anomalous transport (as found for existing experiments) and effect of density peaking on the bootstrap current, $q_{0}$ sustainment and fusion power.

Starting with the current ramp up phase an impact of external heating and current drive waveforms, timing of the L-H transition and plasma current waveform on the target q-profile and resistive flux consumption at the end of the current ramp up have been investigated [42]. It has been shown that the q profile with $q_{0}>1$ can be reached at the end of the current ramp up phase with the heating systems available at ITER. The optimisation of the main heating phase performed first in 0-D simulations with METIS by varying the density profile at fixed line averaged density in high confinement plasmas $\left(H_{98 I P B(y, 2)} \sim 1.4\right)$ with high pedestal pressure (up to $100 \mathrm{kPa}$ ) shows that peaked density profiles are required to reach an important fraction of the bootstrap current (above 30\%) and $Q_{D T}(7-8)$, and to sustain the q-profile above unity. Further assessment of the effect of the density profile peaking in 1-D modelling with the GLF23 and EPED models and optimised heating and current drive mix shows that the bootstrap current fraction of $30 \%$ is achieved with peaked density profile which is the marginal value to maintain the q-profile above unity while the global confinement is $H_{98 I P B(y, 2)}$ 1.05-1.08. Indeed, the EPED model predicts reduced pedestal pressure in the case of reduced pedestal density (i.e. peaked density profile) at $I p=11.5-11.8$ MA limiting the $H_{98 I P B(y, 2)}$ factor close to unity. The possible ways to soften this restriction by operating at higher plasma current (13 MA) or by changing the density peaking at fixed (high) pedestal density could be investigated in future. Another important point to be assessed in future simulations of ITER hybrid scenario is the self-consistent modelling of temperatures and density taking into account the modifications of the transport models matching the existing experiments. In addition to the estimation of the ITER hybrid performance and its margins 
based on the experimentally validated models the ITER modelling database can be used for the development of the integrated model-based plasma control strategy [54]. The first steps towards the automatic plasma control and optimisation have been done by developing the combined magnetic and burn control sharing a common set of dedicated actuators [54-57]. This shows that in a fusion device such as ITER, current profile control can be combined with burn control and open the route towards controlled high performance operation.

Acknowledgement This work, supported by the European Communities under the contracts of Association between EURATOM and CEA, CCFE, IST, FOM, IPP, ÖAW, TEKES was carried out within the framework of the Task Force on Integrated Tokamak Modelling of the European Fusion Development Agreement. The views and opinions expressed herein do not necessarily reflect those of the European Commission. 


\section{References}

[1] Gormezano C et al Plasma Phys. Control. Fusion 46 (2004) B435

[2] Gormezano C. et al Progress in the ITER Physics Basis Chapter 6: Steady State Operation Nucl. Fusion 47 (2007) S285

[3] Joffrin E. Plasma Phys. Control. Fusion 49 (2007) B629

[4] Falchetto G. et al The European Integrated Tokamak Modelling (ITM) effort: achievements and first physics results submitted to Nuclear Fusion (March 2013)

[5] Kalupin D. et al The European Transport Solver: an integrated approach for transport simulations in the plasma core submitted to Nuclear Fusion (March 2013)

[6] G. Giruzzi G. et al in Fusion Energy 2012 (Proc. 24th Int. Conf. San Diego, 2012), paper TH/P2-03 http://www-naweb.iaea.org/napc/physics/FEC/FEC2012/index.htm

[7] Parail V. et al, Nucl. Fusion 49 (2009) 075030

[8] Pereverzev G.V., Yushmanov P.N. 2002 Report IPP 5/98, Max-Planck-Institute fur Plasmaphysik

[9] Artaud J.F. et al, Nucl. Fusion 50 (2010) 043001

[10] Cennacchi G. and Taroni A. 1988 JET-IR(88) 03

[11] Kessel C.E. et al Nucl. Fusion 47 (2007) 1274

[12] Murakami M. et al Nucl. Fusion 51 (2011) 103006

[13] Kritz et al Nucl. Fusion 51 (2011) 123009

[14] Parail V. et al Self-consistent simulation of plasma scenarios for ITER using a combination of $1.5 \mathrm{D}$ transport codes and free boundary equilibrium codes submitted to Nuclear Fusion (March 2013)

[15] ITER Physics Basis Expert Groups on Confinement and Transport and Confinement Modelling and Database, ITER Physics Basis Editors 1999 Nucl. Fusion 392175

[16] Joffrin E et al Nucl. Fusion 45 (2005) 626

[17] Joffrin E et al 2010 Proc. 23rd Int. Conf. on Fusion Energy 2010 (Daejeon, South Korea 2010) (Vienna: IAEA) CD-ROM file EXC/1-1 and http://wwwnaweb.iaea.org/napc/physics/FEC/FEC2010/html/index.htm

[18] Hobirk J et al Plasma Phys. Control. Fusion 54 (2012) 095001

[19] Stober J et al Nucl. Fusion 47 (2007) 728

[20] Garcia J to appear in Plasma Phys. Control. Fusion (2013)

[21] Houlberg W A et al Phys. Plasmas 4 (1997) 3230

[22] Hirshman S P and Sigmar D J Nucl. Fusion 21 (1981) 1079

[23] Shaing K C, Yokoyama M, Wakatani M and Hsu C T Phys. Plasmas 3 (1996) 965

[24] Mc Carthy P.J. et al, Plas. Phys. Control. Fusion 54 (2012) 015100

[25] Lao L L et al Nucl. Fusion 301035 (1990)

[26] Na Y.S et al, Nucl. Fusion 46 (2006) 232

[27] Citrin J. et al, Plasma Phys. Contr. Fusion 54 (2012) 065008

[28] Waltz R.E., Staebler G.M., Dorland W., Hammett G.W., Kotschenreuther M. and Konings J.A. Phys. Plasmas 7 (1997) 2482

[29] Mantica P. et al Phys. Review Letter 107 (2011) 135004

[30] Voitsekhovitch I. et al, Proc. 39th EPS Conference on Plasma Physics and 16th International Congress on Plasma Physics (Stockholm, Sweden, 2012), Vol. 36F file P4.066 (2012) http://ocs.ciemat.es/EPSICPP2012PAP/html/

[31] Voitsekhovitch et al, Nucl. Fusion 49 (2009) 055026

[32] Kinsey J. E., Waltz R. E., and Candy J. Phys. Plasmas 12 (2005) 062302 
[33] Garzotti L et al, Proc. 39th EPS Conference on Plasma Physics and 16th International Congress on Plasma Physics (Stockholm, Sweden, 2012), Vol. 36F file P1.004 (2012) http://ocs.ciemat.es/EPSICPP2012PAP/html/

[34] Politzer P.A. et al., Nucl. Fusion 48 (2008) 075001

[35] Laborde L., McDonald D. C. and Voitsekhovitch I. Phys. Plasmas 15, (2008) 102507

[36] Fundamenski W., Militello F., Moulton D. and McDonald D.C. Nucl. Fusion 52 (2012) 062003

[37] Erba M, Cherubini A, Parail V, Sprigmann E and Taroni A Plasma Phys. Contr. Fusion 39 (1997) 261

[38] Artaud J.F. et al, in proceeding 32nd EPS Conf. on Plasma Phys. and Contr. Fusion, (Spain, Tarragona, 2005) ECA Vol. 29C, P1.035 (2005) http://epsppd.epfl.ch/Tarragona/start.htm

[39] McDonald D.C. et al, Nucl. Fusion 47 (2007) 147

[40] Garcia J. and Giruzzi G. Phys. Rev. Lett. 104 (2010) 205003

[41] Hogeweij G.M.D. et al, in Proc. 37th EPS Conference on Plasma Physics (Dublin, Ireland, 2010), ECA Vol. 34A P1.1041 (2010)

[42] Hogeweij G.M.D. et al, Plasma and Fusion Research 7 (2012) 2403063 http://www.jspf.or.jp/PFR/PFR_articles/pfr2012S1/pfr2012_07-2403063.html

[43] Hogeweij G.M.D. et al, Nucl. Fusion 53 (2013) 013008

[44] Imbeaux F. et al, Nucl. Fusion 51 (2011) 083026

[45] Voitsekhovitch I. et al Plasma Phys. Contr. Fusion 52 (2010) 105011

[46] Ejima S. Nucl. Fusion 22 (1982) 1313

[47] Snyder P. B., Groebner R. J., Leonard A. W., Osborne T. H., and Wilson H. R. Physics of Plasmas 16 (2009) 056118

[48] Snyder P.B. et al Nucl. Fusion 51 (2011)103016

[49] Snyder P.B. et al Phys. Plasmas 19 (2012) 056115

[50] Snyder P.B et al in Proc. 39th EPS Conference on Plasma Physics and 16th International Congress on Plasma Physics (Stockholm, Sweden, 2012), Vol. 36F file P4.029 (2012) http://ocs.ciemat.es/EPSICPP2012PAP/html/

[51] Snyder P. B., Groebner R. J., Leonard A. W., Osborne T. H., and Wilson H. R. Phys. Plasmas 16 (2009) 056118

[52] Citrin J. et al, Nucl. Fusion 50 (2010) 115007

[53] Angioni C. et al Nucl. Fusion 47 (2007) 1326

[54] Moreau D. et al., Nucl. Fusion 48 (2008) 106001

[55] Moreau D. et al., Nucl. Fusion 51 (2011) 063009

[56] Moreau D. et al. Integrated magnetic and kinetic control of advanced tokamak plasmas on DIII-D based on data-driven models to appear Nuclear Fusion (Provisionally scheduled for May 2013)

[57] Liu F. et al., in Proc. 39th EPS Conference on Plasma Physics and 16th International Congress on Plasma Physics (Stockholm, Sweden, 2012), Vol. 36F file P1.063 (2012) http://ocs.ciemat.es/EPSICPP2012PAP/html/ 